\title{
DISTANCE TO UNCONTROLLABILITY FOR CONVEX PROCESSES*
}

\author{
ADRIAN LEWIS ${ }^{\dagger}$, RENÉ HENRION ${ }^{\ddagger}$, AND ALBERTO SEEGER $§$
}

\begin{abstract}
The classical study of controllability of linear systems assumes unconstrained control inputs. The "distance to uncontrollability" measures the size of the smallest perturbation to the matrix description of the system rendering it uncontrollable and is a key measure of system robustness. We extend the standard theory of this measure of controllability to the case where the control input must satisfy given linear inequalities. Specifically, we consider the control of differential inclusions, concentrating on the particular case where the control input takes values in a given convex cone.
\end{abstract}

Key words. convex processes, controllability, rank condition, uncontrollable modes, adjoint processes, cone-constrained controls, distance to uncontrollability

AMS subject classifications. 15A18, 93B05, 47H04, 34A60

DOI. $10.1137 / 050628982$

1. Introduction. Classical linear control theory concerns a system of the form

$$
\dot{x}(t)=A x(t)+B u(t),
$$

where, at each time $t$, the state vector $x(t)$ lies in the space $\mathbb{R}^{n}$, the input control $u(t)$ lies in the space $\mathbb{R}^{m}$, and the given matrices $A$ and $B$ are real and of appropriate dimensions. A key question is controllability - whether $x$ can be steered from the origin to an arbitrary point in the state space. To fix the ideas, suppose the input function $u(\cdot)$ is taken from

$$
\mathcal{U}=\left\{u:[0, T] \rightarrow \mathbb{R}^{m}\left|\int_{0}^{T}\right| u(t) \mid d t<\infty\right\},
$$

the space of integrable functions over a prescribed time interval $[0, T]$. The associated trajectory

$$
t \mapsto x_{u, A, B}(t)=\int_{0}^{t} e^{(t-s) A} B u(s) d s
$$

is then an element of the function space

$$
\mathcal{X}=\left\{x:[0, T] \rightarrow \mathbb{R}^{n} \mid x \text { is absolutely continuous }\right\} .
$$

Controllability of the linear system (1.1), or equivalently of the pair $(A, B)$, simply means that

$$
\left\{x_{u, A, B}(T) \mid u \in \mathcal{U}\right\}=\mathbb{R}^{n} .
$$

\footnotetext{
* Received by the editors April 11, 2005; accepted for publication (in revised form) September 27, 2005; published electronically February 15, 2006.

http://www.siam.org/journals/sicon/45-1/62898.html

${ }^{\dagger}$ Cornell University, School of Operations Research and Industrial Engineering, 234 Rhodes Hall, Ithaca, NY 14853 (aslewis@orie.cornell.edu).

${ }^{\ddagger}$ Weierstrass Institute for Applied Analysis and Stochastics, 10117 Berlin, Germany (henrion@wias-berlin.de)

$\S$ University of Avignon, Department of Mathematics, 33, rue Louis Pasteur, 84000 Avignon, France (alberto.seeger@univ-avignon.fr).
} 
For convenience, we see $(A, B)$ not just as a pair of matrices but also as an element of $\mathcal{L}\left(\mathbb{R}^{n+m}, \mathbb{R}^{n}\right)$, the space of linear maps from $\mathbb{R}^{n+m}$ to $\mathbb{R}^{n}$. Spaces of this type are equipped with the operator (or spectral) norm, which we denote by $\|\cdot\|$. Norms in standard Euclidean spaces are denoted simply by $|\cdot|$. To avoid a possible misunderstanding, let us be more explicit:

$$
\|(A, B)\|=\sup _{|(s, w)|=1}|A s+B w| .
$$

As pointed out by Lee and Markus [25], the set

$$
\Xi=\left\{(A, B) \in \mathcal{L}\left(\mathbb{R}^{n+m}, \mathbb{R}^{n}\right) \mid \text { the system (1.1) is uncontrollable }\right\}
$$

is closed. This fact prompted Paige [27] to introduce the number

$$
\mu(A, B)=\inf _{(C, D) \in \Xi}\|(A, B)-(C, D)\|
$$

as measure for the "degree of controllability" of a given $(A, B)$. The number (1.2) indicates how much we need to perturb the system (1.1) in order to destroy its controllability.

The problem of estimating (1.2) is of importance for control theorists and engineers alike. In section 3 we review what has been done already in connection with the evaluation of Paige's distance function $\mu: \mathcal{L}\left(\mathbb{R}^{n+m}, \mathbb{R}^{n}\right) \rightarrow \mathbb{R}$. We also clarify a point that remained a bit obscure until now, namely, the difference between real and complex controllability.

The purpose of our work is to go beyond the traditional context of the unconstrained linear model (1.1). As shown in section 5, the discussion becomes more involved when the input function $u(\cdot)$ is subject to constraints. New concepts and tools are needed to handle this more general situation. Sections 7 and 8 are devoted to the controllability analysis of dynamical systems described by convex processes.

The notation that we employ is for the most part standard; however, a partial list is provided for the reader's convenience:

$$
\begin{array}{lr}
\operatorname{Im} L=\left\{L s \mid s \in \mathbb{R}^{n}\right\} & \text { (range of an operator } L \text { defined on } \mathbb{R}^{n} \text { ), } \\
\operatorname{Ker} L=\left\{s \in \mathbb{R}^{n} \mid L s=0\right\} & \text { (nullspace of an operator } L \text { defined on } \mathbb{R}^{n} \text { ), } \\
\operatorname{dist}[z, \Gamma]=\inf _{\gamma \in \Gamma}|z-\gamma| & \text { (distance from } z \text { to the set } \Gamma \text { ), } \\
\operatorname{span} K=K-K & \text { (space spanned by the cone } K \subset \mathbb{R}^{n} \text { ), } \\
\operatorname{lin} K=K \cap-K & \text { (lineality space of the cone } K \subset \mathbb{R}^{n} \text { ), } \\
K^{+}=\left\{q \in \mathbb{R}^{n} \mid q^{T} s \geq 0 \forall s \in K\right\} & \text { (dual cone of } K \subset \mathbb{R}^{n} \text { ), } \\
S^{\perp}=\left\{q \in \mathbb{R}^{n} \mid q^{T} s=0 \forall s \in S\right\} & \text { (orthogonal space of } S \subset \mathbb{R}^{n} \text { ), } \\
\operatorname{gr} \mathcal{F}=\left\{(s, v) \in \mathbb{R}^{n} \times \mathbb{R}^{n} \mid v \in \mathcal{F}(s)\right\} & \left(\text { graph of a process } \mathcal{F}: \mathbb{R}^{n} \rightrightarrows \mathbb{R}^{n}\right), \\
\operatorname{dom} \mathcal{F}=\left\{s \in \mathbb{R}^{n} \mid \mathcal{F}(s) \neq \emptyset\right\} & \left(\text { domain of a process } \mathcal{F}: \mathbb{R}^{n} \rightrightarrows \mathbb{R}^{n}\right. \text { ), } \\
\operatorname{Im} \mathcal{F}=\cup_{s \in \mathbb{R}^{n}} \mathcal{F}(s) & \left(\text { image of a process } \mathcal{F}: \mathbb{R}^{n} \rightrightarrows \mathbb{R}^{n}\right) .
\end{array}
$$


2. The reduction lemma. Controllability is a linear-algebraic property of the matrix pair $(A, B)$, and in this framework, the problem of computing the distance to uncontrollability is a matrix distance problem. As is often the case for such problems, rank-one perturbations are important. We capture the essential idea in the following abstract linear algebra result that plays a ubiquitous role throughout this work. The notation $x^{T}$ indicates the transpose of the column vector $x$.

Lemma 2.1 (reduction lemma). Let $\Gamma \subset \mathbb{R}^{p}$ be a nonempty set, $x \in \mathbb{R}^{n}$ a nonzero vector, $y \in \mathbb{R}^{p}$, and $F \in \mathcal{L}\left(\mathbb{R}^{n}, \mathbb{R}^{p}\right)$. Then,

$$
\inf _{\substack{E \in \mathcal{L}\left(\mathbb{R}^{n}, \mathbb{R}^{p}\right) \\ E x-y \in \Gamma}}\|E-F\|=\frac{1}{|x|} \operatorname{dist}[F x-y, \Gamma] .
$$

Furthermore, if $\gamma$ is a point in $\Gamma$ at minimal distance from $F x-y$, then

$$
E=F+\frac{1}{|x|^{2}}(y-F x+\gamma) x^{T}
$$

achieves the infimum on the left-hand side of (2.1).

Proof. Denote by $\alpha$ the term on the left-hand side of (2.1). Then,

$$
\begin{aligned}
\alpha & =\inf _{\substack{E \in \mathcal{L}\left(\mathbb{R}^{n}, \mathbb{R}^{p}\right) \\
E x-y \in \Gamma}} \sup _{|s|=1}|(E-F) s| \geq \inf _{\substack{E \in \mathcal{L}\left(\mathbb{R}^{n}, \mathbb{R}^{p}\right) \\
E x-y \in \Gamma}}\left|(E-F)\left(\frac{x}{|x|}\right)\right| \\
& \geq \frac{1}{|x|} \inf _{\substack{E \in \mathcal{L}\left(\mathbb{R}^{n}, \mathbb{R}^{p}\right) \\
E x-y \in \Gamma}}|(E x-y)-(F x-y)| \geq \frac{1}{|x|} \operatorname{dist}[F x-y, \Gamma] .
\end{aligned}
$$

To prove the reverse inequality, we find a sequence $\left\{\gamma_{\nu}\right\}_{\nu \in \mathbb{N}}$ in $\Gamma$ such that

$$
\left|F x-y-\gamma_{\nu}\right| \leq \operatorname{dist}[F x-y, \Gamma]+\nu^{-1} \quad \forall \nu \in \mathbb{N} .
$$

(Recall that $\Gamma$ is not assumed to be closed.) The corresponding linear map

$$
E_{\nu}=F+\frac{1}{|x|^{2}}\left(y-F x+\gamma_{\nu}\right) x^{T}
$$

satisfies $E_{\nu} x-y \in \Gamma$, and therefore

$$
\begin{aligned}
\alpha & \leq\left\|E_{\nu}-F\right\|=\frac{1}{|x|^{2}} \sup _{|s|=1}\left|\left(y-F x+\gamma_{\nu}\right) x^{T} s\right| \\
& \leq \frac{\left|y-F x+\gamma_{\nu}\right|}{|x|} \leq \frac{\operatorname{dist}[F x-y, \Gamma]+\nu^{-1}}{|x|} .
\end{aligned}
$$

We now let $\nu \rightarrow \infty$ and arrive at the desired conclusion. The second part of the lemma is obtained by working with $\gamma$ instead of the minimizing sequence $\left\{\gamma_{\nu}\right\}_{\nu \in \mathbb{N}}$.

What formula (2.1) says is that our complicated approximation problem in the space $\left(\mathcal{L}\left(\mathbb{R}^{n}, \mathbb{R}^{p}\right),\|\cdot\|\right)$ can be reduced to a simpler approximation problem over the Euclidean space $\left(\mathbb{R}^{p},|\cdot|\right)$.

3. The unconstrained linear model. Paige's measure of controllability (1.2), while rather natural, is not the most amenable to analysis. We therefore begin our exposition by discussing the easier case first analyzed by Paige, allowing the pair $(A, B)$ to have complex entries. Most of the material presented in this section is well known, 
but we take the opportunity to clarify some common points of confusion. The original Paige measure of controllability is the distance function $\mu_{\text {complex }}: \mathcal{L}\left(\mathbb{C}^{n+m}, \mathbb{C}^{n}\right) \rightarrow \mathbb{R}$ defined by

$$
\mu_{\text {complex }}(A, B)=\inf _{\substack{(C, D) \text { uncontrollable } \\(C, D) \in \mathcal{L}\left(\mathbb{C}^{n+m}, \mathbb{C}^{n}\right)}}\|(A, B)-(C, D)\| .
$$

A celebrated result due to Eising [9] asserts that

$$
\mu_{\text {complex }}(A, B)=\inf _{z \in \mathbb{C}} \sigma_{\min }[A-z I, B],
$$

where the term on the right-hand side concerns the minimization of the smallest singular value of the rectangular matrix $[A-z I, B]$ with respect to the complex scalar $z$. This minimization problem has been extensively studied in the last years (cf. $[6,10,11,14,17]$ ), so we don't indulge in this matter. Suffice it to say that the Eising formula has its root in the Hautus [16] characterization of controllability:

$$
(A, B) \text { is controllable } \Longleftrightarrow \operatorname{rank}[A-z I, B]=n \quad \forall z \in \mathbb{C} .
$$

We would like to stress the fact that in this paper we are going to work with control systems described only in terms of real entries. The field of complex numbers is ill adjusted when it comes to conically constrained control systems or, more generally, with convex processes.

As shown by Gracia and de Hoyos [15], even if $(A, B)$ has real entries, the uncontrollable $(C, D)$ achieving the infimum in (3.1) may well have complex entries. The "real" Paige function (1.2) is not just the restriction of $\mu_{\text {complex }}$ to the real field. The question of estimating the real Paige function can be answered in at least two different ways.

3.1. The approach of DeCarlo and Wicks. In what follows, we identify the set

$$
\mathcal{O}(r, n)=\left\{Q \in \mathcal{L}\left(\mathbb{R}^{r}, \mathbb{R}^{n}\right) \mid Q^{T} Q=I\right\}
$$

with the collection of orthonormal matrices of size $n \times r$. The following variational formula involves a minimization over the collection of orthonormal matrices having at most two columns.

Proposition 3.1 (see DeCarlo and Wicks [8]). Consider a controllable operator $(A, B) \in \mathcal{L}\left(\mathbb{R}^{n+m}, \mathbb{R}^{n}\right)$. Then, one has

$$
\mu(A, B)=\inf _{Q \in \mathcal{O}(1, n) \cup \mathcal{O}(2, n)}\left\|\left(Q^{T} A\left(I-Q Q^{T}\right), Q^{T} B\right)\right\| .
$$

From a computational point of view, formula (3.3) is not very satisfactory because it involves a minimization problem over a complicated set of matrices. Notice that (3.3) can be written in the form

$$
\mu(A, B)=\min \left\{\mu_{1}(A, B), \mu_{2}(A, B)\right\},
$$

where the term

$$
\begin{aligned}
\mu_{1}(A, B) & =\inf _{Q \in \mathcal{O}(1, n)}\left\|\left(Q^{T} A\left(I-Q Q^{T}\right), Q^{T} B\right)\right\|=\inf _{|q|=1}\left[\left|\left(I-q q^{T}\right) A^{T} q\right|^{2}+\left|B^{T} q\right|^{2}\right]^{1 / 2} \\
& =\inf _{|q|=1} \inf _{\lambda \in \mathbb{R}}\left[\left|A^{T} q-\lambda q\right|^{2}+\left|B^{T} q\right|^{2}\right]^{1 / 2}=\inf _{\lambda \in \mathbb{R}} \sigma_{\min }[A-\lambda I, B]
\end{aligned}
$$


is rather easy to evaluate, but the computation of

$$
\mu_{2}(A, B)=\inf _{Q \in \mathcal{O}(2, n)}\left\|\left(Q^{T} A\left(I-Q Q^{T}\right), Q^{T} B\right)\right\|
$$

remains a difficult task. As observed in [15], the term $\mu_{1}(A, B)$ is not necessarily equal to $\mu(A, B)$. As a general rule, it is only an upper bound.

3.2. The approach of $\mathbf{H u}$ and Davison. An alternative formula for estimating the real Paige function has been suggested by $\mathrm{Hu}$ and Davison [19, 20]. In the proposition stated below, the symbols $\mathcal{R} W$ and $\mathcal{I} W$ refer, respectively, to the real part and the imaginary part of a complex linear map $W \in \mathcal{L}\left(\mathbb{C}^{n+m}, \mathbb{C}^{n}\right)$. The notation $\operatorname{sssv}(E)$ stands for the second-smallest singular value of the matrix $E$.

Proposition 3.2 (see Hu and Davison $[19,20]$ ). Consider a controllable operator $(A, B) \in \mathcal{L}\left(\mathbb{R}^{n+m}, \mathbb{R}^{n}\right)$. Then,

$$
\mu(A, B)=\inf _{z \in \mathbb{C}} \sup _{\gamma \in] 0,1]} \operatorname{sssv}\left(\left[\begin{array}{cc}
\mathcal{R} W_{z} & -\gamma \mathcal{I} W_{z} \\
\gamma^{-1} \mathcal{I} W_{z} & \mathcal{R} W_{z}
\end{array}\right]\right)
$$

with $W_{z}=[A-z I, B]$.

Paradoxically, the evaluation of the real Paige function is much more involved than the evaluation of the complex counterpart. This should not be very surprising, however, for readers who have encountered a similar phenomenon while comparing the real stability radius of a matrix to the complex one. (See the survey paper of Hinrichsen and Pritchard [18].)

3.3. Partial perturbations. The case of perturbations in the pair $(A, B)$ is the most popular one, but other situations could be considered as well. It may happen, for instance, that only the component $A$ is subject to perturbations. The partial index

$$
\partial_{A} \mu(A, B)=\inf _{\substack{C \in \mathcal{L}\left(\mathbb{R}^{n}, \mathbb{R}^{n}\right) \\(C, B) \text { uncontrollable }}}\|A-C\|
$$

indicates how much one needs to perturb the first component of $(A, B)$ in order to produce a pair which is uncontrollable. A similar interpretation must be given to the number

$$
\partial_{B} \mu(A, B)=\inf _{\substack{D \in \mathcal{L}\left(\mathbb{R}^{m}, \mathbb{R}^{n}\right) \\(A, D) \text { uncontrollable }}}\|B-D\|
$$

Later on, these indices are used in the more general context of cone-constrained linear systems (section 5) and control systems governed by convex processes (section 7).

4. Incorporating linear constraints on the input function. Our aim in this work is to extend the classical theory of the distance to uncontrollability to the case where the control $u$ is constrained. As a first, easy but illuminating, step, let us consider the case of linear equality constraints. The works of DeCarlo and Wicks [8] and $\mathrm{Hu}$ and Davison $[19,20]$ can both be extended to the case of a linear system with linear constraints on the input function:

$$
\left\{\begin{aligned}
\dot{x}(t) & =A x(t)+B u(t) \\
u(t) & \in S
\end{aligned}\right.
$$


Controllability for the model (4.1) simply means that $\left\{x_{u, A, B}(T) \mid u \in \mathcal{U}_{S}\right\}=\mathbb{R}^{n}$, with

$$
\mathcal{U}_{S}=\{u \in \mathcal{U} \mid u(t) \in S \text { a.e. on }[0, T]\}
$$

For convenience, we introduce the notation

$$
\Xi(S)=\left\{(A, B) \in \mathcal{L}\left(\mathbb{R}^{n+m}, \mathbb{R}^{n}\right) \mid \text { the system (4.1) is uncontrollable }\right\} .
$$

TheOREM 4.1 (transfer theorem). Let $S$ be an $r$-dimensional subspace of $\mathbb{R}^{m}$. Then, the index of controllability

$$
\mu_{S}(A, B)=\inf _{(C, D) \in \Xi(S)}\|(A, B)-(C, D)\|
$$

for the model (4.1) is given simply by

$$
\mu_{S}(A, B)=\mu(A, B Q),
$$

where $Q \in \mathcal{L}\left(\mathbb{R}^{r}, \mathbb{R}^{m}\right)$ is any orthonormal map having $S$ as range.

Proof. The subspace $S$ can be represented as the range of a certain orthonormal map $Q \in \mathcal{L}\left(\mathbb{R}^{r}, \mathbb{R}^{m}\right)$. By writing the input $u$ in the form $u(t)=Q w(t)$, we arrive at a linear control problem

$$
\dot{x}(t)=A x(t)+B Q w(t)
$$

where the input function $w$ is chosen without restrictions. It is not difficult to see that (4.1) is controllable if and only if the pair $(A, B Q)$ is controllable. This simple but important fact is at the origin of formula (4.3). First, one can write

$$
\|(A, B)-(C, D)\| \geq\|(A, B Q)-(C, D Q)\| \quad \forall(C, D) \in \mathcal{L}\left(\mathbb{R}^{n+m}, \mathbb{R}^{n}\right)
$$

because $Q$ is orthonormal. Thus,

$$
\begin{aligned}
\mu_{S}(A, B) \geq \inf _{\substack{C, D) \in \mathcal{L}\left(\mathbb{R}^{n+m}, \mathbb{R}^{n}\right) \\
(C, D Q) \text { uncontrollable }}}\|(A, B Q)-(C, D Q)\| \\
\geq \inf _{\substack{(C, Y) \in \mathcal{L}\left(\mathbb{R}^{n+r}, \mathbb{R}^{n}\right) \\
(C, Y) \text { uncontrollable }}}\|(A, B Q)-(C, Y)\|=\mu(A, B Q) .
\end{aligned}
$$

For the proof of the reverse inequality $\mu_{S}(A, B) \leq \mu(A, B Q)$, pick up any solution $\left(C^{*}, Y^{*}\right)$ to the minimization problem

$$
\left\{\begin{array}{l}
\operatorname{minimize}\|(A, B Q)-(C, Y)\| \\
(C, Y) \in \mathcal{L}\left(\mathbb{R}^{n+r}, \mathbb{R}^{n}\right) \text { uncontrollable. }
\end{array}\right.
$$

Since the map $D \in \mathcal{L}\left(\mathbb{R}^{m}, \mathbb{R}^{n}\right) \mapsto D Q \in \mathcal{L}\left(\mathbb{R}^{r}, \mathbb{R}^{n}\right)$ is surjective, one can write

$$
\mu(A, B Q)=\inf _{\substack{D \in \mathcal{L}\left(\mathbb{R}^{m}, \mathbb{R}^{n}\right) \\ D Q=Y^{*}}}\left\|(A, B Q)-\left(C^{*}, D Q\right)\right\| .
$$

We now construct a $D^{*} \in \mathcal{L}\left(\mathbb{R}^{m}, \mathbb{R}^{n}\right)$ such that

$$
D^{*} Q=Y^{*} \text { and }\left\|(A, B Q)-\left(C^{*}, D^{*} Q\right)\right\|=\left\|(A, B)-\left(C^{*}, D^{*}\right)\right\| .
$$


To see that this is possible, take an orthonormal map $V \in \mathcal{L}\left(\mathbb{R}^{m-r}, \mathbb{R}^{m}\right)$ such that $\operatorname{Im} V=S^{\perp}$, and define

$$
D^{*}=Y^{*} Q^{T}+B V V^{T} .
$$

With this particular choice, one has

$$
\begin{aligned}
& D^{*} Q=Y^{*} Q^{T} Q+B V V^{T} Q=Y^{*}, \\
& D^{*} V=Y^{*} Q^{T} V+B V V^{T} V=B V .
\end{aligned}
$$

Hence,

$$
\begin{aligned}
& \left\|(A, B)-\left(C^{*}, D^{*}\right)\right\|=\sup _{|(s, w)| \leq 1}\left|\left(A-C^{*}\right) s+\left(B-D^{*}\right) w\right| \\
= & \sup _{\left|\left(s, \gamma_{1}, \gamma_{2}\right)\right| \leq 1}\left|\left(A-C^{*}\right) s+\left(B-D^{*}\right)\left(Q \gamma_{1}+V \gamma_{2}\right)\right| \\
= & \sup _{\left|\left(s, \gamma_{1}\right)\right| \leq 1}\left|\left(A-C^{*}\right) s+\left(B-D^{*}\right) Q \gamma_{1}\right|=\left\|(A, B Q)-\left(C^{*}, D^{*} Q\right)\right\| .
\end{aligned}
$$

Notice that $\left(C^{*}, D^{*}\right) \in \Xi(S)$. The combination of (4.6) and (4.7) produces then the desired inequality, completing the proof in this way.

Remark. The proof technique of the transfer theorem tells us, in fact, how to construct an operator $\left(C^{*}, D^{*}\right)$ achieving the infimum (4.2) in the definition of $\mu_{S}(A, B)$. Everything boils down to solving the easier and well-understood minimization problem (4.5).

We end this section with a proposition concerning the partial indices

$$
\begin{gathered}
\partial_{A} \mu_{S}(A, B)=\inf _{\substack{C \in \mathcal{L}\left(\mathbb{R}^{n}, \mathbb{R}^{n}\right) \\
(C, B) \in \Xi(S)}}\|A-C\|, \\
\partial_{B} \mu_{S}(A, B)=\inf _{\substack{D \in \mathcal{L}\left(\mathbb{R}^{m}, \mathbb{R}^{n}\right) \\
(A, D) \in \Xi(S)}}\|B-D\| .
\end{gathered}
$$

As was done in the transfer theorem, it is possible to get rid again of the linear contraint set $S$.

Proposition 4.2. Suppose that $S$ is an r-dimensional subspace of $\mathbb{R}^{m}$ and that $Q \in \mathcal{L}\left(\mathbb{R}^{r}, \mathbb{R}^{m}\right)$ is an orthonormal map having $S$ as range. Then,

$$
\partial_{A} \mu_{S}(A, B)=\partial_{A} \mu(A, B Q) \quad \text { and } \quad \partial_{B} \mu_{S}(A, B)=\partial_{B} \mu(A, B Q) .
$$

Proof. We take into account the transformation $u(t)=Q w(t)$ that leads to the unconstrained control system (4.4). One can show straightforwardly the first equality in (4.8), as well as

$$
\partial_{B} \mu_{S}(A, B) \geq \inf _{\substack{D \in \mathcal{L}\left(\mathbb{R}^{m}, \mathbb{R}^{n}\right) \\(A, D Q) \text { uncontrollable }}}\|B Q-D Q\| \geq \partial_{B} \mu(A, B Q) .
$$

For the proof of the reverse inequality $\partial_{B} \mu_{S}(A, B) \leq \partial_{B} \mu(A, B Q)$, pick up any solution $Y^{*}$ to the problem

$$
\left\{\begin{array}{l}
\text { minimize }\|B Q-Y\| \text { with respect to } \\
Y \in \mathcal{L}\left(\mathbb{R}^{r}, \mathbb{R}^{n}\right) \text { such that }(A, Y) \text { is uncontrollable, }
\end{array}\right.
$$


and observe that

$$
\partial_{B} \mu(A, B Q)=\inf _{\substack{D \in \mathcal{L}\left(\mathbb{R}^{m}, \mathbb{R}^{n}\right) \\ D Q=Y^{*}}}\|B Q-D Q\|
$$

It suffices then to construct a $D^{*} \in \mathcal{L}\left(\mathbb{R}^{m}, \mathbb{R}^{n}\right)$ such that

$$
D^{*} Q=Y^{*} \quad \text { and } \quad\left\|B Q-D^{*} Q\right\|=\left\|B-D^{*}\right\| .
$$

The construction of $D^{*}$ and the remaining part of the proof is as in Theorem 4.1.

5. The cone-constrained linear model. In the previous section we saw that restricting controls to take values in a subspace presents no substantial technical difficulties to the classical theory of controllability. In this section we take the next natural step: conical constraints. The problem of controlling a linear system by using positive inputs has been recognized as an important one since the pioneering works of Brammer [5] and Korobov [22] (see also Son [32]).

5.1. Preliminaries. The model under consideration in this section is

$$
\left\{\begin{aligned}
\dot{x}(t) & =A x(t)+B u(t), \\
u(t) & \in P
\end{aligned}\right.
$$

where the closed convex cone $P$ is regarded as the set of "positive" elements in $\mathbb{R}^{m}$. (Typically, $P$ is the positive orthant of $\mathbb{R}^{m}$.)

Controllability for the model (5.1) is defined in a similar way as before, except that now the contraint set is not the subspace $S$ but the cone $P$. Controllability of (5.1) implies, of course, controllability of the relaxed control problem

$$
\left\{\begin{aligned}
\dot{x}(t) & =A x(t)+B u(t) \\
u(t) & \in \operatorname{span} P .
\end{aligned}\right.
$$

Relaxation is a convenient device to be back in a linear setting, where simple and nice controllability tests are available. In what follows, we use the notation

$$
\ll A, B, P \gg=B(P)+A B(P)+\cdots+A^{n-1} B(P),
$$

where addition of sets is understood in the usual Minkowski sense, and powers of $A \in \mathcal{L}\left(\mathbb{R}^{n}, \mathbb{R}^{n}\right)$ correspond to iterated compositions. Since $P$ is a convex cone, the set $\ll A, B, P \gg$ is also a convex cone and

$$
\operatorname{span} \ll A, B, P \gg=\ll A, B, \operatorname{span} P \gg=\left\{\mathcal{C}^{A, B} \gamma: \gamma \in[\operatorname{span} P]^{n}\right\}
$$

with $\mathcal{C}^{A, B}=\left[B, A B, \ldots, A^{n-1} B\right]$ denoting the controllability matrix associated to the pair $(A, B)$. If one represents the space $\operatorname{span} P$ as the range of a linear map $Q \in \mathcal{L}\left(\mathbb{R}^{r}, \mathbb{R}^{m}\right)$, with $r=\operatorname{dim}[\operatorname{span} P]$, then

$$
\operatorname{span} \ll A, B, P \gg=\operatorname{Im} \mathcal{C}^{A, B Q} .
$$

Proposition 5.1. The following three conditions are equivalent:

(i) the relaxed system (5.2) is controllable,

(ii) $\ll A, B, P \gg$ spans the whole space $\mathbb{R}^{n}$,

(iii) $\mathcal{C}^{A, B Q}$ has full rank. 
Proof. This result is surely well known since it is an obvious extension of Kalman's controllability theorem [21].

Unfortunately, the relaxation (or linearization) mechanism $P \mapsto \operatorname{span} P$ destroys part of the information contained in the original model (5.1). For recovering the information that is lost, we introduce the concept of "unilateral uncontrollable mode."

Definition 5.2. One says that $\lambda \in \mathbb{R}$ is an uncontrollable mode of $(A, B)$ relative to $P$ if

$$
\operatorname{Im}(A-\lambda I)+B(P) \neq \mathbb{R}^{n} .
$$

Such an uncontrollable mode $\lambda$ is declared unilateral if $\operatorname{Im}(A-\lambda I)+B(P)$ has nonempty interior; otherwise it is declared bilateral.

If the relaxed system (5.2) is controllable, then we should not worry about the existence of uncontrollable modes of the bilateral type. In fact, one has the next lemma.

Lemma 5.3. Suppose that $\ll A, B, P \gg$ spans $\mathbb{R}^{n}$. Then, $(A, B)$ doesn't have bilateral uncontrollable modes relative to $P$.

Proof. This corresponds to a particular case of a more general result stated in section 7, namely, Proposition 7.9.

That $\ll A, B, P \gg$ spans $\mathbb{R}^{n}$ doesn't rule out, however, the existence of uncontrollable modes of the unilateral type. This is an important point that deserves to be stressed.

THEOREM 5.4. Controllability of the cone-constrained linear model (5.1) is equivalent to the combination of the following two conditions:

(i) $\ll A, B, P \gg$ spans $\mathbb{R}^{n}$,

(ii) $(A, B)$ has no unilateral uncontrollable mode relative to $P$.

Proof. According to Brammer [5], controllability of (5.1) is equivalent to the combination of (i) and

$$
\left\{\begin{array}{l}
\text { the matrix } A^{T} \text { has no (real) eigenvalue with } \\
\text { associated eigenvector in the cone }[B(P)]^{+} .
\end{array}\right.
$$

Since

$$
[B(P)]^{+}=\left\{q \in \mathbb{R}^{n} \mid B^{T} q \in P^{+}\right\},
$$

Brammer's condition (5.4) is just another way of saying that $(A, B)$ has no uncontrollable mode relative to $P$. Due to Lemma 5.3, bilateral uncontrollable modes can be taken out of the discussion. Indeed, these modes are excluded by the property (i).

5.2. Divide and conquer. As shown in the above theorem, controllability of a cone-constrained linear model is a concept that can be broken into two different pieces. The first piece is a sort of generalized Kalman's rank condition. It takes into account the span of the cone $P$, but not the cone itself. This condition is purely linear in the sense that it doesn't recognize the "conic" part of $P$. The second piece takes care of the possible gap between the cone $P$ and its span. In line with this observation, we split the set

$$
\Xi(P)=\left\{(A, B) \in \mathcal{L}\left(\mathbb{R}^{n+m}, \mathbb{R}^{n}\right) \mid \text { the system (5.1) is uncontrollable }\right\}
$$


in two different components:

$$
\begin{aligned}
& \Xi^{\text {rank }}(P)=\left\{(A, B) \in \mathcal{L}\left(\mathbb{R}^{n+m}, \mathbb{R}^{n}\right) \mid \operatorname{span} \ll A, B, P \gg \neq \mathbb{R}^{n}\right\}, \\
& \Xi^{\text {uni }}(P)=\left\{(A, B) \in \mathcal{L}\left(\mathbb{R}^{n+m}, \mathbb{R}^{n}\right) \mid \sigma_{P}^{\text {uni }}(A, B) \neq \emptyset\right\} .
\end{aligned}
$$

The notation $\sigma_{P}^{\text {uni }}(A, B)$ refers, of course, to the set of all unilateral uncontrollable modes of $(A, B)$ relative to $P$. Since

$$
\Xi(P)=\Xi^{\mathrm{rank}}(P) \cup \Xi^{\mathrm{uni}}(P),
$$

the index of controllability

$$
\mu_{P}(A, B)=\inf _{(C, D) \in \Xi(P)}\|(A, B)-(C, D)\|
$$

for the cone-constrained model (5.1) can be computed by using the rule

$$
\mu_{P}(A, B)=\min \left\{\mu_{P}^{\mathrm{rank}}(A, B), \mu_{P}^{\mathrm{uni}}(A, B)\right\}
$$

where the component indices $\mu_{P}^{\mathrm{rank}}(A, B)$ and $\mu_{P}^{\mathrm{uni}}(A, B)$ are defined in an obvious manner.

The evaluation of $\mu_{P}^{\text {rank }}$ is the "easy" part of the job. What we have to do is to adjust $\mathrm{Hu}$-Davison's formula to the linearly constrained control system (5.2).

Proposition 5.5. Let $P$ be an $r$-dimensional closed convex cone in $\mathbb{R}^{m}$. Let $Q \in \mathcal{L}\left(\mathbb{R}^{r}, \mathbb{R}^{m}\right)$ be any orthonormal map such that span $P=\operatorname{Im} Q$. Consider an operator $(A, B) \in \mathcal{L}\left(\mathbb{R}^{n+m}, \mathbb{R}^{n}\right)$ such that $\ll A, B, P \gg$ spans $\mathbb{R}^{n}$. Then,

$$
\mu_{P}^{\mathrm{rank}}(A, B)=\inf _{z \in \mathbb{C}} \sup _{\gamma \in] 0,1]} \operatorname{sssv}\left(\left[\begin{array}{cc}
\mathcal{R} W_{z} & -\gamma \mathcal{I} W_{z} \\
\gamma^{-1} \mathcal{I} W_{z} & \mathcal{R} W_{z}
\end{array}\right]\right)
$$

with $W_{z}=[A-z I, B Q]$.

Proof. By definition, $\mu_{P}^{\mathrm{rank}}$ is the distance function to the set $\Xi^{\mathrm{rank}}(P)$. Since

$$
\mu_{P}^{\mathrm{rank}}(A, B)=\mu_{\mathrm{span} P}(A, B),
$$

it suffices to combine Theorem 4.1 and Proposition 3.2.

The evaluation of $\mu_{P}^{\text {uni }}$ falls beyond the context of $\mathrm{Hu}-$ Davison's formula. We no longer seem able to use arguments in the realm of standard linear algebra. The number

$$
\mu_{P}^{\text {uni }}(A, B)=\inf _{(C, D) \in \Xi_{\text {uni }}(P)}\|(A, B)-(C, D)\|
$$

indicates how much we need to perturb the pair $(A, B)$ if we wish to produce a unilateral uncontrollable mode.

Before trying to compute this number, let us say a few additional words on the set $\Xi^{\text {uni }}(P)$. In the very definition of this set, we use implicitly the expression

$$
P^{\oplus}=P^{+} \backslash \operatorname{lin}\left(P^{+}\right) .
$$

We don't know if there is already a name for $P^{\oplus}$, so we call it the pseudo-dual cone of $P$. Without loss of generality we may suppose that $P$ is not a subspace. If $P$ were a subspace, then $P^{\oplus}$ would be empty, and $\Xi^{\text {uni }}(P)$ would be empty as well. Observe that the cone $P^{\oplus}$ is convex but not necessarily closed. 
Lemma 5.6. Consider a closed convex cone $K$ in some arbitrary Euclidean space. If $K$ is not a subspace, then one has $\operatorname{cl}[K \backslash \operatorname{lin} K]=K$ and also $\operatorname{cl}\left[K^{\oplus}\right]=K^{+}$.

Proof. We prove only $K \subset \operatorname{cl}[K \backslash \operatorname{lin} K]$, the reverse inclusion being trivial. Take $c \in K$. Suppose that $c \in \operatorname{lin}(K)$; otherwise we are done. Since $K$ is not a subspace, we can pick up some $c^{*} \in K \backslash \operatorname{lin} K$ and form

$$
\left.c_{\alpha}=(1-\alpha) c+\alpha c^{*} \quad \text { with } \quad \alpha \in\right] 0,1[.
$$

Since $c$ and $c^{*}$ are in $K$, so is the convex combination $c_{\alpha}$. The equality

$$
c^{*}=\alpha^{-1} c_{\alpha}-\alpha^{-1}(1-\alpha) c
$$

implies that $c_{\alpha}$ doesn't belong to $\operatorname{lin}(K)$. Hence, $c_{\alpha} \in K \backslash \operatorname{lin} K$. The desired conclusion is obtained by letting $\alpha \rightarrow 0^{+}$.

Lemma 5.7. Suppose that $P$ is a closed convex cone but not a subspace. Then,

$$
(C, D) \in \Xi^{\text {uni }}(P) \Longleftrightarrow\left\{\begin{array}{l}
\text { one can find } \lambda \in \mathbb{R} \text { and a unit vector } q \\
\text { such that } C^{T} q=\lambda q \text { and } D^{T} q \in P^{\oplus} .
\end{array}\right.
$$

Proof. The proof is not difficult, and therefore it is omitted.

Proposition 5.8. Suppose that $P$ is a closed convex cone but not a subspace. Then, the index (5.6) admits the characterization

$$
\mu_{P}^{\mathrm{uni}}(A, B)=\inf _{\substack{\lambda \in \mathbb{R} \\|q|=1}}\left[\left|A^{T} q-\lambda q\right|^{2}+\operatorname{dist}^{2}\left[B^{T} q, P^{+}\right]\right]^{1 / 2} .
$$

Proof. By using Lemma 5.7, one gets

$$
\mu_{P}^{\text {uni }}(A, B)=\inf _{(C, D)} \inf _{\substack{\lambda \in \mathbb{R},|q|=1 \\ C^{T} q=\lambda q, D^{T} q \in P}}\|(A, B)-(C, D)\|=\inf _{\lambda \in \mathbb{R},|q|=1} \Psi_{A, B}(\lambda, q)
$$

with

$$
\Psi_{A, B}(\lambda, q)=\inf _{\substack{(C, D) \in \mathcal{L}\left(\mathbb{R}^{n+m}, \mathbb{R}^{n}\right) \\ C^{T} q=\lambda q, D^{T} q \in P^{\oplus}}}\|(A, B)-(C, D)\| .
$$

A simple matter of computation shows that

$$
\begin{aligned}
\Psi_{A, B}(\lambda, q)= & \inf _{\substack{(C, D) \in \mathcal{L}\left(\mathbb{R}^{n+m}, \mathbb{R}^{n}\right) \\
\left(C^{T}-\lambda I\right) q=0, D^{T} q \in P^{\oplus}}}\left\|\left[\begin{array}{c}
C^{T}-\lambda I \\
D^{T}
\end{array}\right]-\left[\begin{array}{c}
A^{T}-\lambda I \\
B^{T}
\end{array}\right]\right\| \\
= & \inf _{X, Y}\left\|\left[\begin{array}{c}
X \\
Y
\end{array}\right]-\left[\begin{array}{c}
A^{T}-\lambda I \\
B^{T}
\end{array}\right]\right\|,
\end{aligned}
$$

where the last infimum is taken with respect to

$$
\left[\begin{array}{c}
X \\
Y
\end{array}\right] \in \mathcal{L}\left(\mathbb{R}^{n}, \mathbb{R}^{n+m}\right) \quad \text { such that }\left[\begin{array}{c}
X \\
Y
\end{array}\right] q \in\left[\begin{array}{c}
0 \\
P^{\oplus}
\end{array}\right]
$$

The reduction lemma yields

$$
\Psi_{A, B}(\lambda, q)=\operatorname{dist}\left[\left[\begin{array}{c}
A^{T}-\lambda I \\
B^{T}
\end{array}\right] q,\left[\begin{array}{c}
0 \\
P^{\oplus}
\end{array}\right]\right],
$$

and therefore

$$
\mu_{P}^{\mathrm{uni}}(A, B)=\inf _{\substack{\lambda \in \mathbb{R} \\|q|=1}}\left[\left|A^{T} q-\lambda q\right|^{2}+\operatorname{dist}^{2}\left[B^{T} q, P^{\oplus}\right]\right]^{1 / 2} .
$$

But, due to Lemma 5.6, one can change $P^{\oplus}$ by $P^{+}$. 
6. Partial perturbations of cone-constrained linear models. Analogously to our earlier discussion of partial perturbations, we might wish to consider perturbing only the matrix $A$ in measuring the distance to uncontrollability of the cone-contrained linear model (5.1). The techniques of the previous section extend in a straightforward manner. The nonnegative real number

$$
\partial_{A} \mu_{P}^{\mathrm{uni}}(A, B)=\inf _{\substack{C \in \mathcal{L}\left(\mathbb{R}^{n}, \mathbb{R}^{n}\right) \\ \sigma_{P}^{u n i}(C, B) \neq \emptyset}}\|A-C\|
$$

indicates how much one needs to perturb the first component of $(A, B)$ in order to produce a unilateral uncontrollable mode relative to $P$. A similar interpretation must be given to the number

$$
\partial_{B} \mu_{P}^{\text {uni }}(A, B)=\inf _{\substack{D \in \mathcal{L}\left(\mathbb{R}^{m}, \mathbb{R}^{n}\right) \\ \sigma_{P}^{u n i}(A, D) \neq \emptyset}}\|B-D\| .
$$

In the next proposition we provide the reader with a recipe for computing these partial indices. Then,

Proposition 6.1. Suppose that $P$ is a closed convex cone but not a subspace.

$$
\partial_{A} \mu_{P}^{\mathrm{uni}}(A, B)=\inf _{\substack{\lambda \in \mathbb{R},|q|=1 \\ B^{T} q \in P^{+}}}\left|A^{T} q-\lambda q\right|
$$

and

$$
\partial_{B} \mu_{P}^{\mathrm{uni}}(A, B)=\inf _{\substack{\lambda \in \mathbb{R},|q|=1 \\ A^{T} q=\lambda q}} \operatorname{dist}\left[B^{T} q, P^{+}\right]
$$

Proof. Both formulas are obtained by employing a similar proof technique as in Proposition 5.8. By way of example, let us write

$$
\partial_{A} \mu_{P}^{\text {uni }}(A, B)=\inf _{C \in \mathcal{L}\left(\mathbb{R}^{n}, \mathbb{R}^{n}\right)} \inf _{\substack{\lambda \in \mathbb{R},|q|=1 \\ C^{T} q=\lambda q, q \in[B(P)]^{\oplus}}}\|A-C\|=\inf _{\substack{\lambda \in \mathbb{R},|q|=1 \\ q \in[B(P)]^{\oplus}}} \Psi_{A}(\lambda, q)
$$

with

$$
\Psi_{A}(\lambda, q)=\inf _{\substack{C \in \mathcal{L}\left(\mathbb{R}^{n}, \mathbb{R}^{n}\right) \\ C^{T} q=\lambda q}}\|A-C\|=\inf _{\substack{X \in \mathcal{L}\left(\mathbb{R}^{n}, \mathbb{R}^{n}\right) \\ X q=0}}\left\|X-\left(A^{T}-\lambda I\right)\right\|=\left|A^{T} q-\lambda q\right| .
$$

The last equality is obtained, of course, by applying the reduction lemma. Notice that due to Lemma 5.6 and a continuity argument, the last infimum in (6.3) can be written with $[B(P)]^{+}$instead of $[B(P)]^{\oplus}$.

The partial indices $\partial_{A} \mu_{P}^{\mathrm{rank}}$ and $\partial_{B} \mu_{P}^{\mathrm{rank}}$ are defined in an obvious manner:

$$
\partial_{A} \mu_{P}^{\mathrm{rank}}(A, B)=\partial_{A} \mu_{\mathrm{span} P}(A, B)=\inf _{\substack{C \in \mathcal{L}\left(\mathbb{R}^{n}, \mathbb{R}^{n}\right) \\ \ll C, B, \operatorname{span} P \gg \ngtr \mathbb{R}^{n}}}\|A-C\|,
$$

The computation of these indices can be carried out with the help of the transfer formulas established in Proposition 4.2. 
7. Controllability of convex processes. We can consider the control models we have studied so far in a slightly different light, as controlling differential inclusions of the form $\dot{x} \in A x+K$ for convex cones $K$. In the model (5.1), for example, $K=B P$. In this section we broaden this perspective, considering the controllability of a differential inclusion

$$
\dot{x}(t) \in \mathcal{F}(x(t))
$$

whose right-hand side is a strict closed convex processes $\mathcal{F}: \mathbb{R}^{n} \rightrightarrows \mathbb{R}^{n}$. That $\mathcal{F}$ is a closed convex process simply means that

$$
\operatorname{gr} \mathcal{F}=\left\{(s, v) \in \mathbb{R}^{n} \times \mathbb{R}^{n} \mid v \in \mathcal{F}(s)\right\}
$$

is a closed convex cone. Saying that $\mathcal{F}$ is strict is a short way of indicating that $\mathcal{F}$ is nonempty-valued everywhere, that is to say, $\mathcal{F}(s) \neq \emptyset$ for any $s \in \mathbb{R}^{n}$.

DeFinition 7.1. A strict convex process $\mathcal{F}: \mathbb{R}^{n} \rightrightarrows \mathbb{R}^{n}$ is said to be controllable if the corresponding reachable set

$$
\operatorname{Reach}(\mathcal{F})=\{x(T) \mid x \in \mathcal{X} \text { solves (7.1) and } x(0)=0\}
$$

is the whole space $\mathbb{R}^{n}$.

7.1. Characterizing controllability. We know exactly what controllability of $\mathcal{F}$ means in terms of the trajectories of its associated differential inclusion, but it would be helpful to have at our disposal some simple algebraic criteria for checking this property. This topic has been handled in a brilliant manner by Aubin, Frankowska and Olech in their 1986 paper [3]. Their contribution admits, however, a certain number of improvements. To put everything in the right perspective, let us start by recalling two algebraic concepts for an arbitrary convex process. The first concept emerges as an extension of the classical rank condition of Kalman.

Definition 7.2. A convex process $\mathcal{F}: \mathbb{R}^{n} \rightrightarrows \mathbb{R}^{n}$ is said to be reproducing if

$$
\text { there is an integer } k \geq 1 \text { such that } \mathcal{F}^{k}(0) \text { spans } \mathbb{R}^{n},
$$

where the $k$ th power $\mathcal{F}^{k}=\mathcal{F} \circ \cdots \circ \mathcal{F}$ ( $k$-fold) is understood as an iterated composition in the multivalued sense.

We shall say some extra words on the reproducibility or rank condition (7.2) in a moment. The second concept is an extension of Definition 5.2.

DeFinition 7.3. The number $\lambda \in \mathbb{R}$ is called an uncontrollable mode of the convex process $\mathcal{F}: \mathbb{R}^{n} \rightrightarrows \mathbb{R}^{n}$ if $\mathcal{F}-\lambda I$ is not surjective, that is, if $\operatorname{Im}[\mathcal{F}-\lambda I] \neq \mathbb{R}^{n}$. The set of uncontrollable modes of $\mathcal{F}$ is denoted by $\sigma(\mathcal{F})$.

These are the basic ingredients to state the following theorem.

Theorem 7.4 (see Aubin, Frankowska, and Olech, [3]). Let $\mathcal{F}: \mathbb{R}^{n} \rightrightarrows \mathbb{R}^{n}$ be a strict closed convex process. Then,

$\mathcal{F}$ is controllable $\Longleftrightarrow \mathcal{F}$ is reproducing and has no uncontrollable modes.

We mention two ways of rendering this beautiful result even more attractive. First, there is a simple way to characterize the reproducibility condition (7.2). The proposition stated below seems to be new, so we prove it in detail. We rely on two auxiliary lemmas. 
Lemma 7.5. Consider a strict convex process $\mathcal{F}: \mathbb{R}^{n} \rightrightarrows \mathbb{R}^{n}$ and an integer $k \geq 1$. If the spans of the cones $\mathcal{F}^{k-1}(0)$ and $\mathcal{F}^{k}(0)$ coincide, then so do the spans of the cones $\mathcal{F}^{j}(0)$ for exponents $j=k-1, k, k+1, \ldots$

Proof. By induction, it suffices to prove the case $j=k+1$. In this case, if the result fails, there is a vector $v$ in the cone $\mathcal{F}^{k+1}(0)$ outside the span of the cone $\mathcal{F}^{k}(0)$. Choose a vector $s$ in $\mathcal{F}^{k}(0)$ with $v \in \mathcal{F}(s)$ and a vector $w$ in the relative interior of the cone $\mathcal{F}^{k-1}(0)$. Since $s$ lies in the span of the cone $\mathcal{F}^{k-1}(0)$, the vector $b w+s$ lies in $\mathcal{F}^{k-1}(0)$ for some real $b>0$ sufficiently large. Since $\mathcal{F}$ is strict, there is a vector $z$ in $\mathcal{F}(w)$ (and hence in $\mathcal{F}^{k}(0)$ ). Since the graph of $\mathcal{F}$ is a convex cone, the vector $b z+v$ lies in $\mathcal{F}(b w+s)$, and hence in $\mathcal{F}^{k}(0)$, contradicting the fact that $v$ lies outside the span of $\mathcal{F}^{k}(0)$.

Lemma 7.6. Suppose that $\mathcal{F}: \mathbb{R}^{n} \rightrightarrows \mathbb{R}^{n}$ is a strict convex process. Then the interiors of the cones $\mathcal{F}^{k}(0)$ (for exponents $k=n, n+1, \ldots$ ) are either all empty or all nonempty.

Proof. The spans of the cones $\mathcal{F}^{k}(0)$ (for exponents $k=1,2, \ldots$ ) are an increasing sequence of linear subspaces. The previous result implies that equality of two successive elements of the sequence entails constancy thereafter. Hence, by counting dimension, the sequence is constant after at most $n$ elements. The result now follows, since a convex cone has nonempty interior if and only if it spans the whole space.

Remark. One can construct an easy example showing that $\mathcal{F}^{n+1}(0)$ need not be equal to $\mathcal{F}^{n}(0)$. Consider, for instance, $n=2$ and a convex process $\mathcal{F}: \mathbb{R}^{2} \rightarrow \mathbb{R}^{2}$ of the form $\mathcal{F}(s)=A s+K$, with

$$
A=\left[\begin{array}{cc}
\cos \theta & \sin \theta \\
-\sin \theta & \cos \theta
\end{array}\right], K=\mathbb{R}_{+}\left[\begin{array}{l}
1 \\
0
\end{array}\right]
$$

The angle $\theta>0$ is chosen small enough. Since $K$ is a ray and $A$ is a rotation matrix, the set

$$
\mathcal{F}^{k}(0)=K+A(K)+\cdots+A^{k-1}(K)
$$

reduces to the convex cone generated by the vectors

$$
\left[\begin{array}{l}
1 \\
0
\end{array}\right],\left[\begin{array}{c}
\cos ((k-1) \theta) \\
\sin ((k-1) \theta)
\end{array}\right]
$$

This happens as long as $(k-1) \theta \leq \pi$, that is, $k \leq 1+\pi / \theta$. It is only after $k>1+\pi / \theta$ that $\mathcal{F}^{k}(0)=\mathbb{R}^{2}$ becomes constant. Observe that $1+\pi / \theta$ goes to infinity as $\theta \rightarrow 0^{+}$, so one can adjust this example to cover the case of an arbitrary power $n$.

Proposition 7.7. A strict convex process $\mathcal{F}: \mathbb{R}^{n} \rightrightarrows \mathbb{R}^{n}$ is reproducing if and only if $\mathcal{F}^{n}(0)$ spans $\mathbb{R}^{n}$.

Proof. This follows from the last lemma.

The second improvement in the presentation of Theorem 7.4 has to do with the nature of uncontrollable modes. The elements of $\sigma(\mathcal{F})$ can be partioned into two different categories. One says that $\lambda \in \sigma(\mathcal{F})$ is of the unilateral type if $\operatorname{Im}[\mathcal{F}-\lambda I]$ has nonempty interior; otherwise, it is declared of the bilateral type. In short, one has a partition

$$
\sigma(\mathcal{F})=\sigma^{\mathrm{uni}}(\mathcal{F}) \cup \sigma^{\mathrm{bi}}(\mathcal{F})
$$

where the notation is self-explanatory. 
The different types of uncontrollability modes are perhaps better understood if we characterize them in terms of the adjoint process of $F$. Recall that the adjoint (or transpose) of the convex process $\mathcal{F}: \mathbb{R}^{n} \rightrightarrows \mathbb{R}^{n}$ is the convex process $\mathcal{F}^{*}: \mathbb{R}^{n} \rightrightarrows \mathbb{R}^{n}$ defined by

$$
\operatorname{gr} \mathcal{F}^{*}=\left\{(q, p) \in \mathbb{R}^{n} \times \mathbb{R}^{n} \mid(-p, q) \in[\operatorname{gr} \mathcal{F}]^{+}\right\}
$$

that is,

$$
(q, p) \in \operatorname{gr} \mathcal{F}^{*} \Longleftrightarrow\langle p, s\rangle \leq\langle q, v\rangle \quad \forall(s, v) \in \operatorname{gr} \mathcal{F}
$$

We assume that the reader is familiar with this transposition mechanism [2, 4, 29]. As observed already in Proposition 2.4 of [30], the convex cone $\operatorname{Im}[\mathcal{F}-\lambda I]$ is related to

$$
\left(\mathcal{F}^{*}-\lambda I\right)^{-1}(0)=\left\{q \in \mathbb{R}^{n} \mid \lambda q \in \mathcal{F}^{*}(q)\right\}
$$

by means of the duality formula

$$
\left(\mathcal{F}^{*}-\lambda I\right)^{-1}(0)=[\operatorname{Im}(\mathcal{F}-\lambda I)]^{+} .
$$

As a consequence of (7.3), it is clear that an uncontrollable mode of $\mathcal{F}$ is exactly the same thing as an eigenvalue of $\mathcal{F}^{*}$. In short,

$$
\sigma(\mathcal{F})=\Lambda\left(\mathcal{F}^{*}\right)
$$

with

$$
\Lambda\left(\mathcal{F}^{*}\right)=\left\{\lambda \in \mathbb{R} \mid \lambda q \in \mathcal{F}^{*}(q) \text { for some } q \neq 0\right\}
$$

denoting the (point) spectrum of $\mathcal{F}^{*}$. General information on point spectra of convex processes can be found, for instance, in [1, 23, 24]. For bilateral uncontrollable modes, one has the next lemma.

Lemma 7.8. Consider a convex process $\mathcal{F}: \mathbb{R}^{n} \rightrightarrows \mathbb{R}^{n}$. For $\lambda \in \mathbb{R}$, the following three conditions are equivalent:

(i) $\lambda$ is a bilateral uncontrollable mode of $\mathcal{F}$,

(ii) the convex cone $\left(\mathcal{F}^{*}-\lambda I\right)^{-1}(0)$ contains a line,

(iii) there is a unit vector $q \in \mathbb{R}^{n}$ such that $\lambda q \in \mathcal{F}^{*}(q)$ and $-\lambda q \in \mathcal{F}^{*}(-q)$.

Proof. The equivalence between (ii) and (iii) is straightforward. The equivalence between (i) and (ii) is again a consequence of the duality formula (7.3).

Remark. A vector $q$ as in Lemma 7.8 (iii) is called a bilateral eigenvector of $\mathcal{F}^{*}$. The concept of bilateral eigenvector is used by Gajardo and Seeger [13] in connection with the asymptotic stability analysis of discrete-time evolution systems governed by convex processes.

Proposition 7.9. For a strict convex process $\mathcal{F}: \mathbb{R}^{n} \rightrightarrows \mathbb{R}^{n}$, one has the implication

$$
\sigma^{\mathrm{bi}}(\mathcal{F}) \neq \emptyset \quad \Longrightarrow \quad \operatorname{int}\left[\mathcal{F}^{k}(0)\right]=\emptyset \quad \forall k \geq 1
$$

In particular, if a strict convex process $\mathcal{F}: \mathbb{R}^{n} \rightrightarrows \mathbb{R}^{n}$ is reproducing, then it has no bilateral uncontrollable modes.

Proof. Take $\lambda \in \sigma^{\mathrm{bi}}(\mathcal{F})$. By Lemma 7.8, there is a unit vector $q \in \mathbb{R}^{n}$ such that

$$
\lambda q \in \mathcal{F}^{*}(q), \quad-\lambda q \in \mathcal{F}^{*}(-q)
$$


We claim that for every $k \in \mathbb{N}$, one has

$$
\lambda^{k} q \in\left(\mathcal{F}^{*}\right)^{k}(q), \quad-\lambda^{k} q \in\left(\mathcal{F}^{*}\right)^{k}(-q) .
$$

The proof is carried out by using an induction argument. The case $k=1$ corresponds to (7.5). Suppose that (7.6) is true for a given $k$, and let us examine the situation for $k+1$. One has

$$
\left(\mathcal{F}^{*}\right)^{k+1}(q)=\mathcal{F}^{*}\left[\left(\mathcal{F}^{*}\right)^{k}(q)\right]=\cup_{z \in\left(\mathcal{F}^{*}\right)^{k}(q)} \mathcal{F}^{*}(z) \supset \mathcal{F}^{*}\left(\lambda^{k} q\right),
$$

and similarly

$$
\left(\mathcal{F}^{*}\right)^{k+1}(-q)=\mathcal{F}^{*}\left[\left(\mathcal{F}^{*}\right)^{k}(-q)\right]=\cup_{z \in\left(\mathcal{F}^{*}\right)^{k}(-q)} \mathcal{F}^{*}(z) \supset \mathcal{F}^{*}\left(-\lambda^{k} q\right) .
$$

We now use the fact that $\mathcal{F}^{*}$ is positively homogeneous. If $\lambda^{k} \geq 0$, then one can write

$$
\lambda^{k} \mathcal{F}^{*}(q) \subset\left(\mathcal{F}^{*}\right)^{k+1}(q), \quad \lambda^{k} \mathcal{F}^{*}(-q) \subset\left(\mathcal{F}^{*}\right)^{k+1}(-q) .
$$

If $\lambda^{k}<0$, then one gets

$$
-\lambda^{k} \mathcal{F}^{*}(-q) \subset\left(\mathcal{F}^{*}\right)^{k+1}(q), \quad-\lambda^{k} \mathcal{F}^{*}(q) \subset\left(\mathcal{F}^{*}\right)^{k+1}(-q) .
$$

In either case, one obtains

$$
\lambda^{k+1} q \in\left(\mathcal{F}^{*}\right)^{k+1}(q), \quad-\lambda^{k+1} q \in\left(\mathcal{F}^{*}\right)^{k+1}(-q),
$$

proving in this way our claim. In fact, we don't use the full power of (7.6). We just observe that

$$
q \in \operatorname{dom}\left(\mathcal{F}^{*}\right)^{k} \cap-\operatorname{dom}\left(\mathcal{F}^{*}\right)^{k},
$$

that is, $\operatorname{dom}\left(\mathcal{F}^{*}\right)^{k}$ is a convex cone containing a line. By invoking the duality formula

$$
\left[\mathcal{F}^{k}(0)\right]^{+}=\operatorname{dom}\left(\mathcal{F}^{*}\right)^{k}
$$

of Phat [28, Prop. 2.5], we conclude that $\mathcal{F}^{k}(0)$ has empty interior.

In view of Propositions 7.7 and 7.9, the Aubin-Frankowska-Olech controllability theorem can be reformulated in the following form.

Corollary 7.10. Suppose that $\mathcal{F}: \mathbb{R}^{n} \rightrightarrows \mathbb{R}^{n}$ is a strict closed convex process. Then,

$$
\mathcal{F} \text { is controllable } \Longleftrightarrow \mathcal{F}^{n}(0) \text { spans } \mathbb{R}^{n} \text { and } \sigma^{\text {uni }}(\mathcal{F}) \text { is empty }
$$

7.2. Checking reproducibility. Deviating momentarily from the main stream of the discussion, we make some comments concerning the concept of reproducibility.

The relaxation mechanism (5.2) introduced in section 5.1 can be extended to the framework of a differential inclusion whose right-hand side is a general convex process.

DeFINITION 7.11. The linear relaxation of a convex process $\mathcal{F}: \mathbb{R}^{n} \rightrightarrows \mathbb{R}^{n}$ is defined as the multivalued operator $\mathcal{F}^{\text {rel }}: \mathbb{R}^{n} \rightrightarrows \mathbb{R}^{n}$ whose graph is given by

$$
\operatorname{gr} \mathcal{F}^{\text {rel }}=\operatorname{gr} \mathcal{F}-\operatorname{gr} \mathcal{F} .
$$

Said in another way, the graph of $\mathcal{F}^{\text {rel }}$ is the linear subspace spanned by the convex cone gr $\mathcal{F}$. 
A more explicit formula for $\mathcal{F}^{\text {rel }}$ is given by

$$
\mathcal{F}^{\mathrm{rel}}(s)=\bigcup_{s_{2}-s_{1}=s} \mathcal{F}\left(s_{2}\right)-\mathcal{F}\left(s_{1}\right) \quad \forall s \in \mathbb{R}^{n} .
$$

By construction, the multivalued operator $\mathcal{F}^{\text {rel }}$ is linear in the sense that

$$
\mathcal{F}^{\mathrm{rel}}\left(\alpha_{1} s_{1}+\alpha_{2} s_{2}\right)=\alpha_{1} \mathcal{F}^{\mathrm{rel}}\left(s_{1}\right)+\alpha_{2} \mathcal{F}^{\mathrm{rel}}\left(s_{2}\right)
$$

$\forall s_{1}, s_{2} \in \operatorname{dom} \mathcal{F}^{\text {rel }}$ and nonzero $\alpha_{1}, \alpha_{2} \in \mathbb{R}$. (That $\alpha_{1}, \alpha_{2} \in \mathbb{R}$ are nonzero scalars is of importance and should not be neglected.) General information on the theory of linear multivalued operators can be found in the book by Cross [7].

We declare the differential inclusion

$$
\dot{x}(t) \in \mathcal{F}^{\mathrm{rel}}(x(t))
$$

as being the relaxed version of the control model (7.1). As we shall see in the next theorem, reproducibility of $\mathcal{F}$ is equivalent to controllability of (7.8). First, we state the next lemma.

LEMma 7.12. For a strict closed convex process $\mathcal{F}: \mathbb{R}^{n} \rightrightarrows \mathbb{R}^{n}$, one has

(i) $\mathcal{F}\left(s_{2}\right)-\mathcal{F}\left(s_{1}\right) \subset \mathcal{F}\left(s_{2}-s_{1}\right)-\mathcal{F}(0) \quad \forall s_{1}, s_{2} \in \mathbb{R}^{n}$,

(ii) $\mathcal{F}^{\mathrm{rel}}(s) \subset \mathcal{F}(s)-\mathcal{F}(0) \quad \forall s \in \mathbb{R}^{n}$,

(iii) $\mathcal{F}(s) \subset \mathcal{F}^{\text {rel }}(s) \quad \forall s \in \mathbb{R}^{n}$,

(iv) $\left(\mathcal{F}^{\text {rel }}\right)^{k}(0)=\operatorname{span} \mathcal{F}^{k}(0) \quad \forall k \in \mathbb{N}$.

Proof. For proving part (i), take $s_{1}, s_{2} \in \mathbb{R}^{n}$ and $v \in \mathcal{F}\left(s_{2}\right)-\mathcal{F}\left(s_{1}\right)$. Write

$$
v=v_{2}-v_{1} \quad \text { with } \quad v_{1} \in \mathcal{F}\left(s_{1}\right), v_{2} \in \mathcal{F}\left(s_{2}\right) .
$$

By strictness of $\mathcal{F}$, we can find some element $w$ in $\mathcal{F}\left(-s_{1}\right)$. Since the graph of $\mathcal{F}$ is a convex cone, it follows that

$$
v_{1}+w \in \mathcal{F}(0), \quad v_{2}+w \in \mathcal{F}\left(s_{2}-s_{1}\right)
$$

Hence,

$$
v=\left(v_{2}+w\right)-\left(v_{1}+w\right) \in \mathcal{F}\left(s_{2}-s_{1}\right)-\mathcal{F}(0) .
$$

Part (ii) follows immediately from (i) and formula (7.7). Part (iii) is trivial because gr $\mathcal{F} \subset$ gr $\mathcal{F}^{\text {rel }}$. The proof of (iv) is more subtle and is based on an induction argument. For $k=1$, the result is true because the equality

$$
\mathcal{F}^{\mathrm{rel}}(0)=\operatorname{span} \mathcal{F}(0)
$$

is obtained by combining (ii) and (iii). Suppose the announced formula is true for a given $k$. For $k+1$, one gets

$$
\begin{aligned}
& \left(\mathcal{F}^{\mathrm{rel}}\right)^{k+1}(0)=\bigcup_{v \in\left(\mathcal{F}^{\mathrm{rel}}\right)^{k}(0)} \mathcal{F}^{\mathrm{rel}}(v)=\bigcup_{v \in \operatorname{span} \mathcal{F}^{k}(0)} \mathcal{F}^{\mathrm{rel}}(v)=\bigcup_{v_{1}, v_{2} \in \mathcal{F}^{k}(0)} \mathcal{F}^{\mathrm{rel}}\left(v_{2}-v_{1}\right) \\
= & \bigcup_{v_{1}, v_{2} \in \mathcal{F}^{k}(0)}\left\{\mathcal{F}^{\mathrm{rel}}\left(v_{2}\right)-\mathcal{F}^{\mathrm{rel}}\left(v_{1}\right)\right\} \subset \bigcup_{v_{1}, v_{2} \in \mathcal{F}^{k}(0)}\left\{\left[\mathcal{F}\left(v_{2}\right)-\mathcal{F}(0)\right]-\left[\mathcal{F}\left(v_{1}\right)-\mathcal{F}(0)\right]\right\} .
\end{aligned}
$$

Therefore,

$$
\left(\mathcal{F}^{\text {rel }}\right)^{k+1}(0) \subset\left[\mathcal{F}\left(\mathcal{F}^{k}(0)\right)-\mathcal{F}(0)\right]-\left[\mathcal{F}\left(\mathcal{F}^{k}(0)\right)-\mathcal{F}(0)\right]=\operatorname{span} \mathcal{F}^{k+1}(0)+\operatorname{span} \mathcal{F}(0) .
$$


The subspace $\operatorname{span} \mathcal{F}(0)$ can be dropped from the last sum because it is contained in $\operatorname{span} \mathcal{F}^{k+1}(0)$. We have proved in this way the inclusion $\left(\mathcal{F}^{\text {rel }}\right)^{k+1}(0) \subset \operatorname{span} \mathcal{F}^{k+1}(0)$. The reverse inclusion is trivial because the convex cone $\mathcal{F}^{k+1}(0)$ is smaller than the linear space $\left(\mathcal{F}^{\text {rel }}\right)^{k+1}(0)$.

LEMma 7.13. Let $\mathcal{F}: \mathbb{R}^{n} \rightrightarrows \mathbb{R}^{n}$ be a strict closed convex process. Then, $\mathcal{F}^{\text {rel }}$ admits the representation

$$
\mathcal{F}^{\mathrm{rel}}(s)=A^{\mathcal{F}} s+S^{\mathcal{F}} \quad \forall s \in \mathbb{R}^{n},
$$

where $S^{\mathcal{F}}=\operatorname{span} \mathcal{F}(0)$, and $A^{\mathcal{F}} \in \mathcal{L}\left(\mathbb{R}^{n}, \mathbb{R}^{n}\right)$ is defined by

$$
A^{\mathcal{F}} s=\pi^{\mathcal{F}}\left[\mathcal{F}^{\mathrm{rel}}(s)\right]
$$

with $\pi^{\mathcal{F}}: \mathbb{R}^{n} \rightarrow \mathbb{R}^{n}$ denoting the orthogonal projection onto $[\operatorname{span} \mathcal{F}(0)]^{\perp}$.

Proof. It must be observed that $\pi^{\mathcal{F}}\left[\mathcal{F}^{\text {rel }}(s)\right]$ is indeed a singleton. To see this, take $y_{1}, y_{2} \in \pi^{\mathcal{F}}\left[\mathcal{F}^{\text {rel }}(s)\right]$ and write

$$
y_{1}=\pi^{\mathcal{F}}\left(v_{1}\right), \quad y_{2}=\pi^{\mathcal{F}}\left(v_{2}\right) \quad \text { with } v_{1}, v_{2} \in \mathcal{F}^{\mathrm{rel}}(s) .
$$

Hence,

$$
y_{1}-y_{2}=\pi^{\mathcal{F}}\left(v_{1}\right)-\pi^{\mathcal{F}}\left(v_{2}\right)=\pi^{\mathcal{F}}\left(v_{1}-v_{2}\right)=0,
$$

the last equality being due to the fact that

$$
v_{1}-v_{2} \in \mathcal{F}^{\mathrm{rel}}(s)-\mathcal{F}^{\mathrm{rel}}(s)=\mathcal{F}^{\mathrm{rel}}(0)=\operatorname{span} \mathcal{F}(0) .
$$

Checking the linearity of the single-valued operator $A^{\mathcal{F}}$ is essentially a matter of exploiting the linearity of the multivalued operator $\mathcal{F}^{\text {rel }}$. The details are omitted. Finally, we check the representation formula (7.9). Take $s \in \mathbb{R}^{n}$ and $y \in A^{\mathcal{F}} s+S^{\mathcal{F}}$. Thus,

$$
y=q_{1}+\pi^{\mathcal{F}}(v) \quad \text { with } q_{1} \in S^{\mathcal{F}}, v \in \mathcal{F}^{\text {rel }}(s) .
$$

Since $q_{2}=v-\pi^{\mathcal{F}}(v) \in S^{\mathcal{F}}$, it follows that

$$
y=q_{1}-q_{2}+v \in S^{\mathcal{F}}+\mathcal{F}^{\mathrm{rel}}(s)=\mathcal{F}^{\mathrm{rel}}(0)+\mathcal{F}^{\mathrm{rel}}(s) \subset \mathcal{F}^{\mathrm{rel}}(s) .
$$

Conversely, take $y \in \mathcal{F}^{\text {rel }}(s)$. Since $y-\pi^{\mathcal{F}}(y) \in S^{\mathcal{F}}$, it follows that

$$
y=\pi^{\mathcal{F}}(y)+\left[y-\pi^{\mathcal{F}}(y)\right] \in A^{\mathcal{F}} s+S^{\mathcal{F}}
$$

Remark. The operator $A^{\mathcal{F}}$ used to represent $\mathcal{F}^{\text {rel }}$ is not unique. In fact, one has $\mathcal{F}^{\text {rel }}(\cdot)=A(\cdot)+S^{\mathcal{F}}$ for any $A \in \mathcal{L}\left(\mathbb{R}^{n}, \mathbb{R}^{n}\right)$ with $\operatorname{Im}\left(A-A^{\mathcal{F}}\right) \subset S^{\mathcal{F}}$. Such $A$ is called a linear selector of $\mathcal{F}^{\text {rel }}$. We declare $A^{\mathcal{F}}$ to be the standard linear selector of $\mathcal{F}^{\text {rel }}$.

In view of Lemma 7.13, the relaxed version of the differential inclusion (7.1) can be written in the form

$$
\left\{\begin{aligned}
\dot{x}(t) & =A^{\mathcal{F}} x(t)+u(t) \\
u(t) & \in S^{\mathcal{F}}
\end{aligned}\right.
$$

a model that is well understood by now. Such linearly constrained control problem can also be written in the unconstrained form

$$
\left\{\begin{array}{l}
\dot{x}(t)=A^{\mathcal{F}} x(t)+Q w(t) \\
w(t) \in \mathbb{R}^{r}
\end{array}\right.
$$


where $r$ is the dimension of $S^{\mathcal{F}}$, and $Q \in \mathcal{L}\left(\mathbb{R}^{r}, \mathbb{R}^{n}\right)$ is any orthonormal map such that $\operatorname{Im} Q=S^{\mathcal{F}}$.

THEOREM 7.14. For a strict closed convex process $\mathcal{F}: \mathbb{R}^{n} \rightrightarrows \mathbb{R}^{n}$, the following five conditions are equivalent:

(i) $\mathcal{F}$ is reproducing,

(ii) $\mathcal{F}^{\text {rel }}$ is reproducing,

(iii) the system (7.11) is controllable,

(iv) $\left(A^{\mathcal{F}}, Q\right)$ is controllable for some $Q \in \mathcal{O}(r, n)$ such that $\operatorname{Im} Q=S^{\mathcal{F}}$,

(v) $\left(A^{\mathcal{F}}, Q\right)$ is controllable for every $Q \in \mathcal{O}(r, n)$ such that $\operatorname{Im} Q=S^{\mathcal{F}}$.

Proof. The equivalence between (i) and (ii) is a consequence of Lemma 7.12(iv). From the controllability theory of linear systems, we know that the conditions (iii), (iv), and (v) are all equivalent to (ii).

8. Additive versus hybrid perturbations. Equipped with the characterizations of controllability of process differential inclusions we explored in the previous section, we can now return to our central topic of measuring the degree of controllability. Robustness of controllability for a system like (7.1) is a topic that has been studied by Naselli-Ricceri [26], Tuan [33], and Lavilledieu and Seeger [24]. Here we go beyond the qualitative analysis carried out by these authors and focus attention on the quantitative aspect. We want to measure how much we need to perturb the system (7.1) in order to destroy its controllability.

8.1. Additive perturbations. The simplest way to perturb the differential inclusion (7.1) is to add a linear map $L \in \mathcal{L}\left(\mathbb{R}^{n}, \mathbb{R}^{n}\right)$ to the reference or nominal operator $\mathcal{F}$. The perturbed system

$$
\dot{x}(t) \in(\mathcal{F}+L)(x(t))
$$

may no longer be controllable if the perturbation $L$ is too severe. The index

$$
\mu_{\text {add }}(\mathcal{F})=\inf _{\substack{L \in \mathcal{L}\left(\mathbb{R}^{n}, \mathbb{R}^{n}\right) \\ \mathcal{F}+L \text { uncontrollable }}}\|L\|
$$

speaks by itself and doesn't need further explanation. In line with the "divide and conquer" strategy adopted in this work, we write

$$
\mu_{\text {add }}(\mathcal{F})=\min \left\{\mu_{\text {add }}^{\text {rank }}(\mathcal{F}), \mu_{\text {add }}^{\text {uni }}(\mathcal{F})\right\},
$$

where

$$
\mu_{\text {add }}^{\text {rank }}(\mathcal{F})=\inf _{\substack{L \in \mathcal{L}\left(\mathbb{R}^{n}, \mathbb{R}^{n}\right) \\ \mathcal{F}+L \text { irreproducing }}}\|L\|
$$

measures the distance to irreproducibility, and

$$
\mu_{\text {add }}^{\text {uni }}(\mathcal{F})=\inf _{\substack{L \in \mathcal{L}\left(\mathbb{R}^{n}, \mathbb{R}^{n}\right) \\ \sigma^{\text {uni }}(\mathcal{F}+L) \neq \emptyset}}\|L\|
$$

indicates how much we need to perturb $\mathcal{F}$ in order to produce a unilateral uncontrollable mode.

In the proof of the theorem stated below, we use the notation $\mathcal{F} \odot$ to indicate the pseudo-adjoint of the convex process $\mathcal{F}: \mathbb{R}^{n} \rightrightarrows \mathbb{R}^{n}$. By definition, $\mathcal{F} \odot: \mathbb{R}^{n} \rightrightarrows \mathbb{R}^{n}$ is the convex process given by $\operatorname{gr} \mathcal{F}^{\odot}=\operatorname{gr} \mathcal{F}^{*} \backslash \operatorname{lin}\left[\operatorname{gr} \mathcal{F}^{*}\right]$, or, more explicitly,

$$
\mathcal{F}^{\odot}(q)=\mathcal{F}^{*}(q) \backslash-\mathcal{F}^{*}(-q) \quad \forall q \in \mathbb{R}^{n} .
$$


TheOREM 8.1. Suppose that $\mathcal{F}: \mathbb{R}^{n} \rightrightarrows \mathbb{R}^{n}$ is a strict closed convex process. Then,

$$
\mu_{\text {add }}^{\text {uni }}(\mathcal{F})=\left\{\begin{array}{cl}
\inf _{\substack{\lambda \in \mathbb{R} \\
|q|=1}} \operatorname{dist}\left[\lambda q, \mathcal{F}^{*}(q)\right] & \text { if } \text { gr } \mathcal{F} \text { is not a subspace } \\
\infty & \text { otherwise }
\end{array}\right.
$$

On the other hand,

$$
\mu_{\text {add }}^{\text {rank }}(\mathcal{F})=\partial_{A} \mu\left(A^{\mathcal{F}}, Q\right)
$$

with $Q \in \mathcal{O}(r, n)$ such that $\operatorname{Im} Q=\operatorname{span} \mathcal{F}(0)$ and $A^{\mathcal{F}}$ denoting the standard linear selector of $\mathcal{F}^{\text {rel }}$.

Proof. The formula (8.5) is based on the fact that $\sigma^{\mathrm{uni}}(\mathcal{F}+L) \neq \emptyset$ if and only if there exist a scalar $\lambda \in \mathbb{R}$ and a unit vector $q \in \mathbb{R}^{n}$ such that

$$
\left\{\begin{array}{c}
\lambda q \in \mathcal{F}^{*}(q)+L^{T} q \\
-\lambda q \notin \mathcal{F}^{*}(-q)-L^{T} q
\end{array}\right.
$$

The above condition can be written in the more compact form $L^{T} q-\lambda q \in-\mathcal{F} \odot(q)$. Hence,

$$
\mu_{\text {add }}^{\operatorname{uni}}(\mathcal{F})=\inf _{\substack{\lambda \in \mathbb{R} \\|q|=1}} \inf _{\substack{L \in \mathcal{L}\left(\mathbb{R}^{n}, \mathbb{R}^{n}\right) \\ L^{T} q-\lambda q \in-\mathcal{F} \odot(q)}}\|L\| .
$$

By applying the reduction lemma, one obtains

$$
\mu_{\text {add }}^{\text {uni }}(\mathcal{F})=\inf _{\substack{\lambda \in \mathbb{R} \\|q|=1}} \operatorname{dist}\left[\lambda q, \mathcal{F}^{\odot}(q)\right] .
$$

Both terms in (8.7) are equal to $\infty$ if gr $\mathcal{F}$ is a subspace. Suppose then that gr $\mathcal{F}$ is not a subspace. Since $\mathcal{F}$ is a strict closed convex process, it follows that $\mathcal{F}^{*}(0)=\{0\}$. Hence,

$$
\mathcal{F}^{*}(q)+\mathcal{F}^{*}(-q) \subset\{0\}
$$

From this relation, one can see that

$$
\operatorname{dom} \mathcal{F}^{\odot}=\operatorname{dom} \mathcal{F}^{*} \backslash \operatorname{lin}\left[\operatorname{dom} \mathcal{F}^{*}\right] \quad \text { and } \mathcal{F}^{\odot}(q)=\mathcal{F}^{*}(q) \quad \forall q \in \operatorname{dom} \mathcal{F}^{\odot} .
$$

It has to be shown that, for arbitrary $\lambda \in \mathbb{R}$ and unit vector $q \in \operatorname{dom} \mathcal{F}^{*}$, one has

$$
\operatorname{dist}\left[\lambda q, \mathcal{F}^{*}(q)\right] \geq \mu_{\text {add }}^{\text {uni }}(\mathcal{F}) .
$$

To do this, we take into account (8.7) and the following two facts. First, due to Lemma 5.6, every unit vector in $\operatorname{dom} \mathcal{F}^{*}$ can be obtained as limit of a sequence of unit vectors taken from $\operatorname{dom} \mathcal{F}^{*} \backslash \operatorname{lin}\left[\operatorname{dom} \mathcal{F}^{*}\right]$. Second, since $\mathcal{F}$ is strict, $\mathcal{F}^{*}$ maps bounded sets to bounded sets and $\mathcal{F}^{*}$ is single-valued over $\operatorname{lin}\left[\operatorname{dom} \mathcal{F}^{*}\right.$ ] (cf. Corollary 2.5.8 in [2]). As far as (8.6) is concerned, one follows a similar proof technique as in the more general situation discussed in Theorem 8.3.

8.2. Hybrid perturbations. Perturbing a differential inclusion by adding a linear map to the right-hand side is not the most general perturbational scheme that one may consider. In fact, a perturbational scheme of the additive type is poorly suited to deal with a large number of important situations occurring in practice. To 
see this, just think of the particular case of the unconstrained linear control problem (1.1). This model can be represented in terms of the convex process $\mathcal{F}_{A, B}: \mathbb{R}^{n} \rightrightarrows \mathbb{R}^{n}$ given by

$$
\mathcal{F}_{A, B}(s)=A s+\operatorname{Im} B .
$$

By adding a linear map $L \in \mathcal{L}\left(\mathbb{R}^{n}, \mathbb{R}^{n}\right)$ to the convex process $\mathcal{F}_{A, B}$ one recovers a perturbed system

$$
\dot{x}(t)=(A+L) x(t)+B u(t)
$$

for which the $B$ component remains unaffected. A more sophisticated operation must be carried out on $\mathcal{F}_{A, B}$ if one wishes to incorporate perturbations in the $B$ component as well.

The concept of hybrid perturbation is based on the simultaneous use of two linear maps, say, $M \in \mathcal{L}\left(\mathbb{R}^{n}, \mathbb{R}^{n}\right)$ and $L \in \mathcal{L}\left(\mathbb{R}^{n}, \mathbb{R}^{n}\right)$, the first acting in an multiplicative way and the second in a additive way. The new convex process

$$
s \in \mathbb{R}^{n} \mapsto[M \circ \mathcal{F}+L](s)=M(\mathcal{F}(s))+L s
$$

is viewed as a perturbed version of $\mathcal{F}$. Of course, perturbation doesn't occur if one takes $(M, L)=(I, 0)$. All this is for saying that

$$
\mu_{\text {hyb }}(\mathcal{F})=\inf _{\substack{M, L \in \mathcal{L}\left(\mathbb{R}^{n}, \mathbb{R}^{n}\right) \\ M \circ \mathcal{F}+L \text { uncontrollable }}}\|(M, L)-(I, 0)\|
$$

is a reasonable candidate for measuring the degree of controllability of $\mathcal{F}$. Observe, incidentally, that hybrid perturbations preserve the strictness of $\mathcal{F}$.

We follow once more our old habit of thought and decompose (8.8) in the form

$$
\mu_{\text {hyb }}(\mathcal{F})=\min \left\{\mu_{\text {hyb }}^{\text {rank }}(\mathcal{F}), \mu_{\text {hyb }}^{\text {uni }}(\mathcal{F})\right\}
$$

with $\mu_{\text {hyb }}^{\text {rank }}(\mathcal{F})$ and $\mu_{\text {hyb }}^{\text {uni }}(\mathcal{F})$ being defined in an obvious way.

THEOREM 8.2. Suppose that $\mathcal{F}: \mathbb{R}^{n} \rightrightarrows \mathbb{R}^{n}$ is a strict closed convex process. Then, one has

$$
\mu_{\text {hyb }}^{\text {uni }}(\mathcal{F})=\left\{\begin{array}{cl}
\inf _{\substack{\lambda \in \mathbb{R} \\
|q|=1}} \operatorname{dist}\left[(q, \lambda q), \operatorname{gr} \mathcal{F}^{*}\right] & \text { if } \operatorname{gr} \mathcal{F} \text { is not a subspace }, \\
\infty & \text { otherwise. }
\end{array}\right.
$$

Proof. We consider only the case when gr $\mathcal{F}$ is not a subspace, the other case being trivial. For any $M, L \in \mathcal{L}\left(\mathbb{R}^{n}, \mathbb{R}^{n}\right)$, one has $(M \circ \mathcal{F}+L)^{*}=\mathcal{F}^{*} \circ M^{T}+L^{T}$. Hence, $\sigma^{\text {uni }}(M \circ \mathcal{F}+L) \neq \emptyset$ if and only if there exist a scalar $\lambda \in \mathbb{R}$ and a unit vector $q \in \mathbb{R}^{n}$ such that

$$
\left\{\begin{aligned}
\lambda q & \in \mathcal{F}^{*}\left(M^{T} q\right)+L^{T} q \\
-\lambda q & \notin \mathcal{F}^{*}\left(-M^{T} q\right)-L^{T} q
\end{aligned}\right.
$$

This can be written in the form

$$
\left(L^{T} q-\lambda q, M^{T} q\right) \in(\operatorname{gr} \mathcal{F})^{\oplus}
$$

with $(\operatorname{gr} \mathcal{F})^{\oplus}$ denoting the pseudo-dual of the convex cone gr $\mathcal{F}$. Hence,

$$
\mu_{\text {hyb }}^{\text {uni }}(\mathcal{F})=\inf _{\substack{\lambda \in \mathbb{R},|q|=1}} \inf _{\substack{M, L \in \mathcal{L}\left(\mathbb{R}^{n}, \mathbb{R}^{n}\right) \\
\left(L^{T} q-\lambda q, M^{T} q\right) \in(\operatorname{grF} \mathcal{F}) \oplus}}\|(M, L)-(I, 0)\|=\inf _{\substack{\lambda \in \mathbb{R},|q|=1}} \inf _{X, Y}\left\|\left[\begin{array}{c}
X \\
Y
\end{array}\right]-\left[\begin{array}{c}
0 \\
I
\end{array}\right]\right\|,
$$


where the last infimum is taken with respect to

$$
\left[\begin{array}{c}
X \\
Y
\end{array}\right] \in \mathcal{L}\left(\mathbb{R}^{n}, \mathbb{R}^{n+m}\right) \text { such that }\left[\begin{array}{c}
X \\
Y
\end{array}\right] q-\left[\begin{array}{c}
\lambda q \\
0
\end{array}\right] \in(\operatorname{gr} \mathcal{F})^{\oplus} .
$$

The reduction lemma yields

$$
\mu_{\text {hyb }}^{\text {uni }}(\mathcal{F})=\inf _{\substack{\lambda \in \mathbb{R},|q|=1}} \operatorname{dist}\left[(-\lambda q, q),(\operatorname{gr} \mathcal{F})^{\oplus}\right]=\inf _{\substack{\lambda \in \mathbb{R} \\|q|=1}} \operatorname{dist}\left[(-\lambda q, q),(\operatorname{gr} \mathcal{F})^{+}\right],
$$

from which one gets the announced result.

TheOREM 8.3. Suppose that $\mathcal{F}: \mathbb{R}^{n} \rightrightarrows \mathbb{R}^{n}$ is a strict closed convex process. Then,

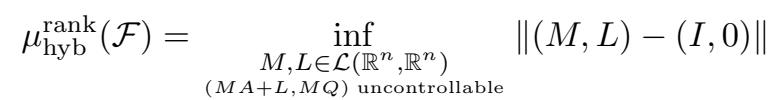

with $Q \in \mathcal{O}(r, n)$ such that $\operatorname{Im} Q=\operatorname{span} \mathcal{F}(0)$ and $A$ denoting any linear selector of $\mathcal{F}^{\text {rel }}$.

Proof. One can show that for any $M, L \in \mathcal{L}\left(\mathbb{R}^{n}, \mathbb{R}^{n}\right)$, one has the identity

$$
\operatorname{gr}(M \circ \mathcal{F}+L)-\operatorname{gr}(M \circ \mathcal{F}+L)=\operatorname{gr}\left(M \circ \mathcal{F}^{\mathrm{rel}}+L\right),
$$

and therefore $(M \circ \mathcal{F}+L)^{\text {rel }}=M \circ \mathcal{F}^{\text {rel }}+L$. By combining this fact and Theorem 7.14 , one sees that

$$
\begin{aligned}
M \circ \mathcal{F}+L \text { is irreproducing } & \Longleftrightarrow(M \circ \mathcal{F}+L)^{\text {rel }} \text { is irreproducing } \\
& \Longleftrightarrow M \circ \mathcal{F}^{\mathrm{rel}}+L \text { is irreproducing } \\
& \Longleftrightarrow(M A+L, M Q) \text { is uncontrollable. }
\end{aligned}
$$

This proves, of course, the announced formula.

We end this section by showing how to evaluate the hybrid indices $\mu_{h y b}^{\mathrm{uni}}$ and $\mu_{h y b}^{\mathrm{rank}}$ in the particular case of a convex process $\mathcal{F}_{A, B}^{P}: \mathbb{R}^{n} \rightrightarrows \mathbb{R}^{n}$ given by

$$
\mathcal{F}_{A, B}^{P}(s)=A s+B(P) .
$$

This choice may seem very peculiar, but, in fact, it is one of the most prominent examples in the general theory of convex processes. Observe that the cone-constrained model (5.1) can be written in the form of a differential inclusion whose right-hand side is $\mathcal{F}_{A, B}^{P}$. For the sake of completeness, we mention that the class

$$
\mathcal{H}_{P}=\left\{\mathcal{F}_{A, B}^{P} \mid(A, B) \in \mathcal{L}\left(\mathbb{R}^{n+m}, \mathbb{R}^{n}\right)\right\}
$$

is stable with respect to hybrid perturbations. Indeed, one can write the identity

$$
M \circ \mathcal{F}_{A, B}^{P}+L=\mathcal{F}_{C, D}^{P},
$$

where the pairs $(A, B)$ and $(C, D)$ are related through the transformation formulas

$$
C=M A+L, \quad D=M B .
$$

Observe that the perturbation $(M, L)$ that brings $(A, B)$ to $(C, D)$ is given by

$$
M=D\left(B^{T} B\right)^{-1} B^{T}, \quad L=C-D\left(B^{T} B\right)^{-1} B^{T} A .
$$


For writing (8.11) we are implicitly assuming that the transpose of $B \in \mathcal{L}\left(\mathbb{R}^{m}, \mathbb{R}^{n}\right)$ is surjective because otherwise $B^{T} B$ is not invertible. In the context of our coneconstrained control problem (5.1), surjectivity of $B^{T}$ can be assumed without loss of generality. It is interesting to note that seemingly more general types of perturbations like pointwise or graphical addition of convex processes do not even allow to recover the class $\mathcal{H}_{p}$ introduced above.

Corollary 8.4. Let $P \subset \mathbb{R}^{m}$ be a closed convex cone but not a subspace. Then,

$$
\mu_{\mathrm{hyb}}^{\mathrm{uni}}\left(\mathcal{F}_{A, B}^{P}\right)=\inf _{\substack{\lambda \in \mathbb{R} \\|q|=1}} \inf _{B^{T} h \in P^{+}}\left[\left|A^{T} h-\lambda q\right|^{2}+|h-q|^{2}\right]^{1 / 2} .
$$

Proof. An easy calculation shows that

$$
\operatorname{gr}\left(\mathcal{F}_{A, B}^{P}\right)^{*}=\left\{(h, p) \mid B^{T} h \in P^{+}, p=A^{T} h\right\} .
$$

It suffices now to apply the general formula (8.9).

We mention in passing that (8.12) can also be obtained by writing

$$
\begin{aligned}
\mu_{\text {hyb }}^{\text {uni }}\left(\mathcal{F}_{A, B}^{P}\right)= & \inf _{\substack{M, L \in \mathcal{L}\left(\mathbb{R}^{n}, \mathbb{R}^{n}\right) \\
\sigma^{u n i}\left(M \circ \mathcal{F}_{A, B}^{P}+L\right) \neq \emptyset}}\|(M, L)-(I, 0)\| \\
= & \inf _{\substack{M, L \in \mathcal{L}\left(\mathbb{R}^{n}, \mathbb{R}^{n}\right), \lambda \in \mathbb{R},|q|=1 \\
A^{T} M^{T} q+L^{T} q=\lambda q, B^{T} M^{T} q \in P^{\oplus}}}\|(M, L)-(I, 0)\|
\end{aligned}
$$

and then applying the reduction lemma. This alternative method, however, requires some additional simplificatory work.

Corollary 8.5. Let $(A, B) \in \mathcal{L}\left(\mathbb{R}^{n+m}, \mathbb{R}^{n}\right)$ and $P \subset \mathbb{R}^{m}$ be a closed convex cone. Take any $Q \in \mathcal{O}(r, n)$ such that $\operatorname{Im} Q=\operatorname{span} B(P)$. Then,

$$
\mu_{\text {hyb }}^{\mathrm{rank}}\left(\mathcal{F}_{A, B}^{P}\right)=\inf _{\substack{M, L \in \mathcal{L}\left(\mathbb{R}^{n}, \mathbb{R}^{n}\right) \\(M A+L, M Q) \text { uncontrollable }}}\|(M, L)-(I, 0)\| .
$$

Proof. It suffices to apply Theorem 8.3, keeping in mind that the relaxed version of $\mathcal{F}_{A, B}^{P}$ is given by $\left[\mathcal{F}_{A, B}^{P}\right]^{\mathrm{rel}}(\cdot)=A(\cdot)+\operatorname{span} B(P)$.

9. By way of conclusion. This paper is about measuring the distance to uncontrollability in cone-constrained linear control problems or, more generally, in control problems described by convex processes. We have adopted the strategy of splitting the analysis into two separate components. One part of our study consists in measuring the distance to irreproducibility. The term reproducibility refers to a suitable generalization of Kalman's rank condition. The second part of our study consists in measuring the distance to unilateral modality (i.e., existence of unilateral uncontrollable modes). It is in this part of our study that the conic aspect of the data (convex cones, convex processes, etc.) comes into the picture. Bilateral uncontrollable modes belong to the realm of classical linear algebra and therefore they are left aside (in fact, they are implicitly incorporated in the analysis of reproducibility).

The formulas for measuring the distance to unilateral modality were obtained by exploiting the reduction lemma. There is a different approach which consists in exploiting the concept of $\varepsilon$-eigenvalue for multivalued operators. Following Gajardo and Seeger [12], we denote by

$$
\Lambda_{\varepsilon}\left(\mathcal{F}^{*}\right)=\left\{\lambda \in \mathbb{R} \mid \exists(q, p) \in \operatorname{gr} \mathcal{F}^{*} \text { with } q \neq 0 \text {, such that }|p-\lambda q| \leq \varepsilon|q|\right\}
$$


the set of $\varepsilon$-eigenvalues of $\mathcal{F}^{*}$. For practical purposes, it is important to estimate the smallest value of $\varepsilon \in \mathbb{R}_{+}$that guarantees the nonvacuity of $\Lambda_{\varepsilon}\left(\mathcal{F}^{*}\right)$. This smallest value is called the spectral threshold of $\mathcal{F}^{*}$. As established in [12], the equality

$$
\inf \left\{\varepsilon \in \mathbb{R}_{+} \mid \Lambda_{\varepsilon}\left(\mathcal{F}^{*}\right) \neq \emptyset\right\}=\inf _{\substack{\lambda \in \mathbb{R} \\|q|=1}} \operatorname{dist}\left[\lambda q, \mathcal{F}^{*}(q)\right]
$$

holds, in particular, when $\mathcal{F}$ is a strict closed convex process. Formula (9.1) gives us an alternative interpretation of the index $\mu_{\text {add }}^{\text {uni }}(\mathcal{F})$ when gr $\mathcal{F}$ is not a subspace (cf. Theorem 8.1).

Remark. When gr $\mathcal{F}$ is not a subspace, both expressions in (9.1) serve not only to measure the distance to unilateral modality but also to modality in general (i.e., existence of uncontrollable modes without specification of their nature). This observation is quite subtle because, in general, the sets $\sigma^{\text {uni }}(\mathcal{F})$ and $\sigma(\mathcal{F})$ don't coincide.

As far as the hybrid index $\mu_{\text {hyb }}^{\text {uni }}(\mathcal{F})$ is concerned, we see now appearing an expression of the form

$$
\Psi_{\mathcal{F}^{*}}(\lambda)=\inf _{|q|=1} \operatorname{dist}\left[(q, \lambda q), g r \mathcal{F}^{*}\right]
$$

which has to be minimized with respect to $\lambda \in \mathbb{R}$. The function $\Psi_{\mathcal{F}^{*}}$ is used by Seeger [31] in connection with the upper stabilization of the point-spectral set-valued mapping $\Lambda$. Observe that in a finite dimensional setting, one has

$$
\Lambda\left(\mathcal{F}^{*}\right)=\left\{\lambda \in \mathbb{R} \mid \Psi_{\mathcal{F}^{*}}(\lambda)=0\right\} .
$$

In an infinite dimensional setting, the above equality is no longer true. As shown in [31], the roots of $\Psi_{\mathcal{F}^{*}}$ produce a set which may be much larger than $\Lambda\left(\mathcal{F}^{*}\right)$ (one gets the so-called approximate or stabilized spectrum of $\mathcal{F}^{*}$ ). This observation is just to warn the reader that some of our results (for instance, Theorem 8.2) do not extend to an infinite dimensional setting, unless important modifications are incorporated. Infinite dimensionality introduces various complications that are not addressed in the present work.

\section{REFERENCES}

[1] F. Alvarez, R. Correa, And P. Gajardo, Inner estimation of the eigenvalue set and exponential series solutions to differential inclusions, J. Convex Anal., 12 (2005), pp. 1-11.

[2] J.P. Aubin And H. Frankowska, Set-Valued Analysis, Birkhäuser, Boston, 1990.

[3] J.P. Aubin, H. Frankowska and C. Olech, Controllability of convex processes, SIAM J. Control Optim., 24 (1986), pp. 1192-1211.

[4] J. Borwein, Adjoint process duality, Math. Oper. Res., 8, (1983), pp. 403-434.

[5] R.F. Brammer, Controllability in linear autonomous systems with positive controllers, SIAM J. Control Optim., 10 (1972), pp. 339-353.

[6] J.V. Burke, A.S. Lewis, and M.L. Overton, Pseudospectral components and the distance to uncontrollability. SIAM J. Matrix Anal. Appl., 26 (2004/05), pp. 350-361.

[7] R. Cross, Multivalued Linear Operators, Marcel Dekker, New York, 1988.

[8] R.A. DeCARlo And M. WiCKs, Computing the distance to an uncontrollable system, IEEE Trans. Automat. Control, 36, (1991), pp. 39-49.

[9] R. Eising, Between controllable and uncontrollable, Systems Control Lett., 4 (1984), pp. 263264.

[10] L. Elsner AND C. HE, An algorithm for computing the distance to uncontrollability, Systems Control Lett., 17 (1991), pp. 453-464.

[11] P. M. Gahinet, Distance to the nearest uncontrollable pair and algebraic Riccati equation, in Proceedings of the 29th Conference on Decision and Control, Honolulu, 1990, pp. 273-278. 
[12] P. Gajardo And A. Seeger, Epsilon-eigenvalues of multivalued operators, Set-Valued Anal., 11 (2003), pp. 273-296.

[13] P. Gajardo And A. Seeger, Higher-Order Spectral Analysis and Weak Asymptotic Stability of Convex Processes, J. Math. Anal. Appl., 2005, in press.

[14] M. Gao And M. Neumann, A global minimum search algorithm for estimating the distance to uncontrollability, Linear Algebra Appl., 188/189 (1993), pp. 305-350.

[15] J.M. Gracia AND I. DE Hoyos, Nearest pair with more nonconstant invariant factors and pseudospectrum, Linear Algebra Appl., 298 (1999), pp. 143-158.

[16] M.L.J. Hautus, Controllability and observability conditions of linear autonomous systems, Nederl. Akad. Wetensch. Proc. Ser. A 72, 31 (1969), pp. 443-448.

[17] C. He, Estimating the distance to uncontrollability: A fast method and a slow one, Systems Control Lett., 26 (1995), pp. 275-281.

[18] D. Hinrichsen and A.J. Pritchard, Real and Complex Stability Radii: A Survey, Progr. Systems Control Theory 6, Birkhäuser, Boston, 1990, pp. 119-162.

[19] G. Hu And E.J. Davison, A real radius measure for controllability, in Proceedings American Control Conference, Arlington, VA, June 2001, pp. 3144-3148.

[20] G. Hu And E.J. DAvison, Real controllability/stabilizability radius of LTI systems, IEEE Trans. Automat. Control, 49 (2004) pp. 254-257.

[21] R. Kalman, Y.C. Ho, and K.S. Narendra, Controllability of linear dynamical systems, Contrib. Differential Equations, 1 (1962), pp. 189-213.

[22] V.I. Korobov, A geometric criterion of local controllability of dynamical systems in the presence of contraints on the control, Differential Equations, 15 (1980), pp. 1136-1142.

[23] P. Lavilledieu And A. Seeger, Existence de valeurs propres pour les systèmes multivoques: résultats anciens et nouveaux, Ann. Sci. Math. Québec, 25 (2001), pp. 47-70.

[24] P. Lavilledieu And A. Seeger, Rank condition and controllability of parametric convex processes, J. Convex Anal., 9 (2002), pp. 535-542.

[25] E.B. Lee and L. Markus, Foundations of Optimal Control Theory, John Wiley, New York, 1967.

[26] O. NASELli-RicCerI, On the controllability of a class of differential inclusions depending on a parameter, J. Optim. Theory Appl., 65 (1990), pp. 281-288.

[27] C.C. PAIGe, Properties of numerical algorithms related to computing controllability, IEEE Automat. Control, 26 (1981), pp. 130-138.

[28] V.N. РнAт, Weak asymptotic stabilizability of discrete-time systems given by set-valued operators, J. Math. Anal. Appl., 202 (1996), pp. 363-378.

[29] R.T. Rockafellar, Convex Analysis, Princeton Academic Press, Princeton, NJ, 1970.

[30] A. SeEger, Spectral analysis of set-valued mappings, Acta Math. Vietnamica, 25 (1998), pp. 4963.

[31] A. SEeger, Stabilization of the point-spectral mapping, J. Nonlinear Convex Anal., 4 (2003), pp. $277-285$.

[32] N.K. Son, Approximate controllability with positive controls, Acta Math. Vietnamica, 22 (1997), pp. 589-620.

[33] H.D. TuAn, On controllability of convex differential inclusions in Banach space, Optimization, 30 (1994), pp. 151-162. 